\title{
On the Performance Analysis of Higher Order QAM Schemes over Mixed RF/FSO Systems
}

\author{
Praveen Kumar Singya ${ }^{1}$, Nagendra Kumar ${ }^{2}$, Vimal Bhatia ${ }^{1}$, Senior Member, IEEE, \\ and Mohamed-Slim Alouini ${ }^{3}$, Fellow, IEEE
}

\begin{abstract}
In this work, the performance of a dual-hop variable gain amplify-and-forward (AF) mixed radio frequency (RF)/free space optics (FSO) system is analyzed in details. As variable gain AF relay network is considered, processing delay results in outdated channel state information (CSI) during amplification at the relay. For general applicability, the RF link is modeled with the generalized Nakagami-m fading. The FSO link is modeled with the Gamma-Gamma distribution which is affected with the atmospheric turbulence and pointing error impairments, and both the intensity modulation with direct detection (IM/DD) and heterodyne detection are employed at the FSO receiver. In this context, analytical expressions of outage probability and ergodic capacity are derived in terms of Meijer-G function and extended generalized bivariate Meijer-G function. For diversity order, asymptotic outage probability expression is also derived. Further, cumulative distribution function based generalized average symbol error rate expressions for various quadrature amplitude modulation (QAM) schemes such as hexagonal QAM, rectangular QAM, and cross QAM are derived in terms of Meijer-G function. Furthermore, a detailed comparative study of various modulation schemes is presented and the impact of pointing error, atmospheric turbulence, outdated CSI, and Nakagami-m parameter are highlighted on the system performance. Finally, all the analytical results are verified through Monte-Carlo simulations.
\end{abstract}

\section{Index Terms}

Nakagami-m, Gamma-Gamma distribution, pointing errors, outdated CSI, ASER, adaptive modulation, hexagonal QAM (HQAM), cross QAM (XQAM), rectangular QAM (RQAM).

\section{INTRODUCTION}

Power efficient high data-rate communication for the future wireless communication systems can be achieved by minimizing the average transmit power for a given bit error rate (BER) or symbol error rate (SER). Typically, higher data-rates for the available bandwidth require high transmit power for the same performance. Adaptive modulation is one such solution which provides spectrally efficient high data-rate transmission and optimum power utilization for the future wireless communication systems [1]. For spectrally efficient high-data rate transmission, higher order modulation techniques such as the family of quadrature amplitude modulations (QAM) (i.e. squared QAM (SQAM), rectangular QAM (RQAM), cross QAM (XQAM), and hexagonal QAM (HQAM)) due to their high power and bandwidth efficiency have received enhanced consideration in present and future wireless communication systems. In SQAM, constellation points are placed at the vertexes of a squared lattice, valid only for the constellations of even power of 2. For better adaptation of given channel conditions, higher flexibility including odd power of 2 constellations can improve the spectral efficiency. Thus, both the even and odd (power of 2) constellations are considered in this work. For odd power of 2 constellations, RQAM is commonly preferred. RQAM is a versatile modulation scheme because orthogonal binary frequency shift keying (OBFSK), quadrature phase shift keying (QPSK), multi-level amplitude shift keying (ASK), and SQAM are its special cases. However, RQAM is not a good choice for odd power of 2 constellations, and instead a modified XQAM constellation is preferred over RQAM due to its reduced peak-toaverage-power ratio (PAPR) and improved power efficiency. XQAM constellations are obtained by removing the outer corner points of RQAM constellations and placing them in a cross shape which minimizes the average power of the constellation [2].

The ever increasing demand for high-data rates further directs towards the formation of an optimum two dimensional (2D) hexagonal shaped constellation which is referred to as HQAM. For a given optimum Euclidean distance between the constellation points, HQAM consists of densest 2D packing which enables improved power efficiency with lower peak and average powers as the constellation size $(M)$ increases. Hence, HQAM provides improved BER/SER performance than the other QAM schemes [2]-[4]. On the basis of trade-off between the detection complexity and power efficiency, the HQAM constellations are further categorized into regular and irregular HQAM structures. Although, regular HQAM has comparatively simpler detection, its power efficiency or BER performance can further be improved for larger values of $M$. The irregular

${ }^{1}$ P. K. Singya and V. Bhatia are with the Discipline of Electrical Engineering, Indian Institute of Technology Indore, Indore-453552, India (e-mail: phd1501102023@iiti.ac.in, vbhatia@iiti.ac.in)

${ }^{2}$ N. Kumar is with the Department of Electronics and Communication Engineering, National Institute of Technology Jamshedpur-831014, India (e-mail: kumar.nagendra86@gmail.com)

${ }^{3}$ M.-S. Alouini is with the Computer, Electrical, and Mathematical Science and Engineering (CEMSE) Division, King Abdullah University of Science and Technology (KAUST), Thuwal 23955-6900, Saudi Arabia (e-mail: slim.alouini@kaust.edu.sa) 
HQAM provides improved power efficiency and optimum performance, however, at the cost of increased detection complexity [1].

Carrier recovery and automatic gain control (AGC) are required when SQAM is opted with differential coding, which are complex to achieve in practice. There were some serious issues with carrier recovery as false locks were observed not only at multiple of $90^{\circ}$, but also at $26+(l \times 90)$ and $52+(l \times 90)$ degrees with $l=0,1,2,3$ [5]. To avoid the use of AGC and to mitigate the false lock problem, star QAM constellation was proposed. Star QAM is a special case of circular amplitude-phase shift keying (APSK), which outperforms the SQAM in peak power limited systems. It consists of multiple concentric PSK circles with equal constellation points in each circle and identical phase angle between them. Amplitude and phase of the constellation points are mutually independent, hence, differential detection can be applied successfully rather than the coherent detection, which omits the need of accurate phase tracking and channel estimation at the receiver. Due to the above favorable features, star QAM is adopted in various satellite communication standards [6], [7] ${ }^{1}$.

In parallel, over the last few years, free space optics (FSO) has tremendously been studied as a substitute to the radio frequency (RF) systems due to its low cost and easy to install system which operates in unlicensed spectrum. FSO also uses lower power and provides larger bandwidth for more number of users as compared to RF. It is a line-of-sight (LoS) technique which provides improved transmission security and immunity from the electromagnetic interference in a given unlicensed spectrum. However, atmospheric turbulence fluctuates both the intensity and phase of the received signal which causes severe performance degradation [8]. Out of the several statistical channel models proposed for the turbulence induced FSO channels, Gamma-Gamma fading model is most commonly preferred which consists of both the small and large scale atmospheric fluctuations. FSO systems are sensitive to weather conditions including fog, rain, aerosols etc. which significantly affects reliability of the FSO systems. The FSO installations are affected by wind loads, earthquakes or thermal expansion which cause the beam to deviate from its original path, thereby resulting in pointing error. Pointing error significantly deteriorates the performance of the FSO link especially when transmitter and receiver apertures are placed on top of high rise buildings. Cooperative relaying has emerged as one of the solutions to counter the turbulence induced fading and increasing the range of FSO systems. Relaying takes the advantage of shorter hops to improve the performance of FSO link as well as coverage of the system. Recently, there has been a growing interest in mixed relay systems consisting of RF and FSO links together to improve the reliability of an FSO network. Further, mixed RF/FSO network can fill up the connectivity gap between the last mile access networks and a backbone network, where maximum possible number of RF signals can be accumulated into single FSO link which allows the system to achieve maximum possible capacity. Since, RF and FSO links operate at different frequency bands, there is no interference between them and hence, RF link can be used in other applications simultaneously when FSO link is communicating in second time phase.

\section{A. Previous Work}

In the literature, many papers on the performance analysis of a dual-hop amplify-and-forward (AF) RF/FSO system under different fading scenarios for both the intensity modulation with direct detection (IM/DD) and heterodyne detection have been reported. In [9], a dual-hop fixed gain asymmetric RF/FSO system with Rayleigh and Gamma-Gamma distributions, respectively for RF and FSO links is considered and exact closed-form expression of outage probability is derived. For the same system model, work is extended in [10] where closed-form expressions of the ergodic capacity, SER, BER, and amount of fading are derived, and the impact of pointing error is observed on them. Outage probability expression for the fixed-gain RF/FSO system with Rayleigh and Málaga distributions, respectively for the RF and FSO links is derived in [11]. In [12], a dual-hop fixed gain RF/FSO system with Nakagami-m and Gamma-Gamma distributions with pointing error, respectively for the RF and FSO links is considered; and closed-form expressions of outage probability, average BER for various modulation schemes, higher order amount of fading, and ergodic capacity are derived. In [13], for a dual-hop fixed gain FSO/RF system with Gamma-Gamma and Nakagami-m distributions, respectively for the FSO and RF links; closed-form expressions of outage probability, ergodic capacity, and average BER are derived in terms of bivariate H-Fox function. In [14], performance of a dual-hop mixed FSO/RF system with energy harvesting is analyzed over pointing error and atmospheric turbulence. For a $\eta-\mu$ and $\mathcal{M}$ distributed mixed RF/FSO system, physical layer security is analyzed in [15] by considering the eavesdropping in RF link. In [16], a dual-hop mixed RF/FSO system with Gamma-Gamma and Nakagami-m distributions, respectively for the FSO and RF links and selection combining at the receiver is considered. Further, co-channel interference at both relay and receiver is considered; outage probability and BER expression for binary modulation schemes are derived. In [17], a dual-hop mixed $\mathrm{RF} / \mathrm{FSO}$ system with $\eta-\mu$ and generalized $\mathcal{K}$ distributions, respectively for the RF and FSO links is considered; closed-form expressions of outage probability, ergodic capacity, and BER for binary modulation schemes are derived. In [18], for a mixed RF/FSO cognitive AF relay network with Nakagami-m and double generalized Gamma distributions, closed-form expressions of outage and asymptotic outage probabilities are only presented.

\footnotetext{
${ }^{1}$ In this work, comparative study of various QAM constellations with a fixed separation of $2 d$ between the constellation points is presented. However, fixed separation of $2 d$ between constellation points is not maintained in star QAM, and hence, its comparison with the other QAM schemes is not presented due to the lack of common platform.
} 
The works presented above consider perfect knowledge of channel state information (CSI) in the RF part which is hardly available in practice. For variable gain AF relay network, relay continuously monitors the RF link and estimates its CSI periodically. During the estimation, an error is occurred as the estimated CSI at relay is not same as it was at the time of transmission, due to the RF channel variations. This imperfect CSI is considered as the outdated CSI at the relay which amplifies it in the second hop. Thus, the impact of outdated CSI is also considered for the performance analysis in the literature. In [19], a fixed-gain dual-hop multi-relay RF/FSO system with Rayleigh and Málaga distributions, respectively for the RF and FSO parts, is considered. Outdated CSI and pointing error are considered in the RF and FSO links, respectively, and the analytical expressions of outage probability and ergodic capacity are derived while the symbol error probability (SEP) for BPSK scheme is obtained in integral form only (no closed-form is provided). In [8] and [20], respectively a variable gain RF/FSO single relay system and fixed-gain RF/FSO multi relay system with Rayleigh and Gamma-Gamma distributions are presented. Outdated CSI in the RF part and pointing error in the FSO part are considered and closed-form expressions of the outage probability and average BER are derived.

In the literature, average SER (ASER) analysis of various higher order QAM schemes over different fading conditions for various relay or non-relay systems have been considered [1]-[3], [21]-[25]. In [1], generalized BER expression for the regular, irregular, and sub-optimum triangular QAM (TQAM) schemes is derived and detection complexity is compared between them. In [2], closed-form ASER expressions of HQAM, RQAM, XQAM, De-QPSK, and $\pi / 4$-QPSK are derived for a multi-relay system over independent and non-identically distributed (i.n.i.d.) Nakagami-m fading links. For a single relay AF network, closed-form expressions for the outage probability, asymptotic outage probability, and ASER of HQAM and RQAM schemes are derived in [3] over i.n.i.d. Nakagami-m fading links with imperfect CSI. In [4], a multi-relay AF system with MRC receiver over i.n.i.d. Nakagami-m fading channels with both integer and non-integer fading parameters is considered in presence of imperfect CSI and non-linear power amplifier at the relay, and analytical expressions of outage probability, asymptotic outage probability, and ASER of HQAM, RQAM, and XQAM schemes are derived. In [21], for a multi-branch non-relay system, exact ASER expressions of RQAM, differentially encoded QPSK (De-QPSK) and $\pi / 4$-QPSK over i.n.i.d. Nakagami-m fading channels are derived. For a non-relay system, generalized SEP expression for HQAM scheme is derived in [23] over Rayleigh fading link. In [26]-[28], ASER performance of various TQAM (or HQAM) constellations is analyzed for non-relaying systems over additive white Gaussian noise (AWGN) channel.

\section{B. Contribution}

To the best of authors' knowledge, performance of futuristic and efficient modulation schemes on dual-hop mixed RF/FSO AF relay system by considering Nakagami-m and Gamma-Gamma distributions for RF and FSO links, respectively has not been considered. Along with this, practical constraint of outdated CSI in RF link and atmospheric turbulence influenced FSO link with pointing error is not analyzed in the literature. Nakagami-m fading is considered to model the RF link due to its generalization to model variety of fading channels with $m \geq 1 / 2$. From this prospective, a variable-gain mixed RF/FSO AF relay system is considered to exploit the benefits of FSO link in non-LoS (NLoS) communication between the end users.

In practice, perfect knowledge of CSI is not available at the receivers. A relay continuously monitors the RF link and estimates its CSI periodically in the first hop. In the second hop, the estimated CSI is outdated during the amplification at the relay. Thus, in the mixed RF/FSO system, impact of the outdated CSI on the overall system performance is also highlighted. This is further exacerbated since CSI is estimated at the receiver. Furthermore, FSO link is influenced with the atmospheric turbulence and pointing error. Hence, study of robustness of higher order modulation schemes in the RF/FSO system with practical constraints is pertinent. The major contributions of this work are:

- Analytical expression of the outage probability is obtained and the impact of pointing error, atmospheric turbulence, outdated CSI, and type of detection are observed on the outage performance.

- Outage probability expressions for the two extreme conditions when there is no correlation $(\rho=0)$ between the actual CSI and outdated CSI, and when both are perfectly correlated $(\rho=1)$ are also obtained. Further, asymptotic outage probability expression is derived at high SNRs to find out the diversity order of the considered system.

- Ergodic capacity expression for the considered system is derived which is expressed in terms of extended generalized bivariate Meijer-G function (EGBMGF). Further, impact of pointing error, atmospheric turbulence, outdated CSI, and type of detection are observed on it.

- Analytical ASER expressions for the higher order QAM techniques (specially HQAM and XQAM) for RF/FSO systems are not available. Further, irregular HQAM constellations after the modifications in the regular HQAM structure are also presented for the optimum SER performance. For this, cumulative distribution function (CDF) based ASER expressions for the general order HQAM, RQAM, and XQAM schemes are derived, and the combined impact of pointing error, atmospheric turbulence, and outdated CSI are observed on the ASER performance.

- For various regular and irregular HQAM constellations, parameters $K, \tau$, and $\tau_{c}$ are presented in Table I. Generalized approximated expressions for $K, \tau$, and $\tau_{c}$ for irregular HQAM scheme in terms of $M$ are presented for the first time. Expressions and values of $K, \tau$, and $\tau_{c}$ for large constellations are given in Table I.

- Comparative study between various QAM schemes for various constellation sizes (up-to 1024-ary) is also presented which concludes irregular HQAM scheme as the optimum modulation scheme. 
The paper is organized as follows. In Section II, the system and channel models are presented. Section III consists of outage probability analysis. Closed-form ergodic capacity expression is presented in Section IV. In Section V, generalized ASER analysis of various QAM schemes is presented. Analytical and simulation results with important insights are presented in Section VI. Finally, paper concludes in Section VII.

\section{System AND Channel Models}

In this work, a dual-hop mixed RF/FSO AF relay system is considered where Nakagami-m and Gamma-Gamma distributions are assumed for the RF and FSO links, respectively. Outdated CSI in RF part and atmospheric turbulence influenced FSO link with pointing error is considered. Transmission of data from source $(S)$ to destination $(D)$ takes place via single relay $(R)$ in two communication phases. In the first phase, received signal at $R$ can be written as

$$
Y_{r}=h_{s r} s+n_{s r},
$$

where $s$ is the transmitted signal with $E\left[|s|^{2}\right]=1$. Further, $h_{s r}$ represents the fading amplitude of the $S-R$ link with $E\left[\left|h_{s r}\right|^{2}\right]=P_{s}$, is considered to be Nakagami-m distributed. Here, $P_{s}$ represents the transmit power at $S$. Also, $n_{s r}$ is the AWGN with 0 mean and $\Omega_{s r}$ variance associated to the $S-R$ link.

In second communication phase, $R$ amplifies the signal received from $S$ with an amplification gain $G$, and forwards it to $D$ via FSO link. Channel parameter of $S-R$ link is estimated at the relay during amplification which is assumed to be outdated. The gain at $R$ can be given as $G^{2}=\frac{1}{\left|\hat{h}_{s r}\right|^{2}+\Omega_{s r}}$, where $\hat{h}_{s r}$ represents the delayed version of $h_{s r}$.

After subcarrier intensity modulation (SIM) application at $R, \mathrm{RF}$ signal is converted into optical signal as

$$
Y_{\text {opt }}=P_{r}\left(1+m_{0} G Y_{r}\right),
$$

where $m_{0}$ represents the modulation index and $P_{r}$ is the average transmitted optical power. Removing the DC bias and converting the optical signal into RF one, signal received at $D$ can be expressed as

$$
Y_{d}=h_{r d} P_{r} G \eta Y_{r}+n_{r d},
$$

where $h_{r d}$ is the fading amplitude of the FSO link which is considered to be Gamma-Gamma distributed. $n_{r d}$ is the AWGN with 0 mean and $\Omega_{r d}$ variance associated to the $R-D$ link. Also, $\eta$ represents the optical-to-electrical conversion coefficient which is assumed to be 1 throughout the analysis. Invoking (1) in (3), we obtain

$$
Y_{d}=P_{r} G \eta h_{s r} h_{r d} x+P_{r} G \eta h_{r d} n_{s r}+n_{r d} .
$$

Substituting the amplification gain $G$ in (4), and considering the medium and high SNR regime, end-to-end (e2e) instantaneous SNR at $D$ can be written as

$$
\gamma=\frac{\gamma_{1} \gamma_{2}}{\hat{\gamma}_{1}+\gamma_{2}}
$$

where $\gamma_{1}=\frac{\left|h_{s r}\right|^{2}}{\Omega_{s r}}, \hat{\gamma}_{1}=\frac{\left|\hat{h}_{s r}\right|^{2}}{\Omega_{s r}}$, and $\gamma_{2}=\frac{\left|h_{r d}\right|^{2} P_{r}^{2} \eta^{2}}{\Omega_{r d}}$.

The RF link $(S-R)$ is characterized with the generalized Nakagami- $m$ fading. Thus, the SNR of $S-D$ link will be Gamma distributed. Hence, the probability density function (PDF) and CDF of its SNR can be given as

$$
\begin{aligned}
& f_{\gamma_{1}}\left(\gamma_{1}\right)=\left[\frac{1}{\Gamma(m)}\left(\frac{m}{\bar{\gamma}_{1}}\right)^{m} \gamma_{1}{ }^{m-1} e^{-\frac{m \gamma_{1}}{\bar{\gamma}_{1}}}\right], \\
& F_{\gamma_{1}}\left(\gamma_{1}\right)=\left[1-\frac{\Gamma\left(m, \frac{m \gamma_{1}}{\bar{\gamma}_{1}}\right)}{\Gamma(m)}\right],
\end{aligned}
$$

respectively, where $\bar{\gamma}(\cdot)$ represents the average SNR of the link. Further, the FSO link $(R-D)$ experiences Gamma-Gamma fading with the pointing error impairment. Thus, generalized PDF and CDF expressions of its SNR for IM/DD or heterodyne detection can be given as [13], [29]

$$
\begin{aligned}
f_{\gamma_{2}}\left(\gamma_{2}\right) & =\frac{\xi^{2}}{i \gamma_{2} \Gamma(\alpha) \Gamma(\beta)} \mathrm{G}_{1,3}^{3,0}\left[\left.\frac{\xi^{2} \alpha \beta}{\xi^{2}+1}\left(\frac{\gamma_{2}}{\Omega_{i}}\right)^{1 / i}\right|_{\xi^{2}, \alpha, \beta} ^{\xi^{2}+1}\right], \\
F_{\gamma_{2}}\left(\gamma_{2}\right) & =A \mathrm{G}_{i+1,3 i+1}^{3 i, 1}\left[\left.\frac{B}{\Omega_{i}} \gamma_{2}\right|_{\mu_{2}, 0} ^{1, \mu_{1}}\right],
\end{aligned}
$$

respectively, where $\mathrm{G}_{p, q}^{m, n}\left[\left.x\right|_{b_{1}, \ldots, b_{q}} ^{a_{1}, \ldots, a_{p}}\right]$ represents the Meijer G-function. Here, $i$ denotes the type of detection i.e. $i=1$ represents heterodyne detection, whereas $i \stackrel{i=}{=} 2$ represents IM/DD detection. $\Omega_{i}$ represents the average SNR of the $R-D$ link for both the detection cases i.e. $\Omega_{1}=\frac{\xi^{2}}{\xi^{2}+1} \bar{\gamma}_{2} \underset{\xi>>1}{=} \bar{\gamma}_{2}$ and $\Omega_{2} \underset{\xi>>1}{=} \bar{\gamma}_{2} \frac{\alpha \beta \xi^{2}\left(\xi^{2}+2\right)}{(\alpha+1)(\beta+1)\left(\xi^{2}+1\right)^{2}}$, where $\xi$ represents the ratio of equivalent beam radius and the pointing error displacement standard deviation (jitter) at the receiver. Further, $A=\frac{i^{\alpha+\beta-2} \xi^{2}}{(2 \pi)^{i-1} \Gamma(\alpha) \Gamma(\beta)}$ and 
$B=\left(\frac{\xi^{2} \alpha \beta}{\left(\xi^{2}+1\right) i^{2}}\right)^{i}$, where $\alpha$ and $\beta$ are the atmospheric turbulence influenced fading/scintillation parameters [13], [30]. Small values of $\alpha$ and $\beta$ correspond to the severe fading conditions. Furthermore, $\mu_{1}=\left(\frac{\xi^{2}+1}{i}, \cdots, \frac{\xi^{2}+i}{i}\right)$ comprises of $i$ terms, and $\mu_{2}=\left(\frac{\xi^{2}}{i}, \cdots, \frac{\xi^{2}+i-1}{i}, \frac{\alpha}{i}, \cdots, \frac{\alpha+i-1}{i}, \frac{\beta}{i}, \cdots, \frac{\beta+i-1}{i}\right)$ comprises of $3 i$ terms.

\section{Outage Probability}

Outage probability is defined as the probability that the e2e SNR of the considered system reaches below a predefined threshold $\left(\gamma_{t h}\right)$, and is given as

$$
\begin{aligned}
\mathcal{P}_{o}\left(\gamma_{t h}\right) & =\mathcal{P}\left(\frac{\gamma_{1} \gamma_{2}}{\hat{\gamma}_{1}+\gamma_{2}} \leq \gamma_{t h}\right) \\
& =1-\int_{0}^{\infty} \int_{0}^{\infty} \mathcal{P}\left(\gamma_{2} \geq \frac{\gamma_{t h} y}{x}\right) f_{\gamma_{1}, \hat{\gamma}_{1}}\left(x+\gamma_{t h}, y\right) d x d y \\
& =1-\int_{0}^{\infty} \int_{0}^{\infty} \bar{F}_{\gamma_{2}}\left(\frac{\gamma_{t h} y}{x}\right) f_{\gamma_{1}, \hat{\gamma}_{1}}\left(x+\gamma_{t h}, y\right) d x d y
\end{aligned}
$$

where $\bar{F}(\cdot)$ represents the complementary CDF (CCDF). Further, $f_{\gamma_{1}, \hat{\gamma}_{1}}(x, y)$ represents the joint PDF of the SNR of $S-R$ link with its delayed replica which can be given as [31], [32]

$$
f_{\gamma_{1}, \hat{\gamma}_{1}}(x, y)=\left(\frac{m}{\bar{\gamma}_{1}}\right)^{m+1} \frac{1}{(1-\rho) \Gamma(m)}\left(\frac{x y}{\rho}\right)^{\frac{m-1}{2}} e^{-\left(\frac{x+y}{1-\rho}\right) \frac{m}{\bar{\gamma}_{1}}} I_{m-1}\left(\frac{2 m \sqrt{\rho x y}}{\bar{\gamma}_{1}(1-\rho)}\right),
$$

where $\rho$ represents the correlation coefficient and $I_{v}(\cdot)$ represents the $v$ th order modified Bessel function of the first kind. Substituting values from (7) and (9) into (8), we get

$$
\begin{aligned}
\mathcal{P}_{o}\left(\gamma_{t h}\right) & =1-\int_{0}^{\infty} \int_{0}^{\infty}\left[1-A \mathrm{G}_{i+1,3 i+1}^{3 i, 1}\left[\left.\frac{B \gamma_{t h}}{\Omega_{i}} \frac{y}{x}\right|_{\mu_{2}, 0} ^{1, \mu_{1}}\right]\right] \\
& \times\left[( \frac { m } { \overline { \gamma } _ { 1 } } ) ^ { m + 1 } \frac { 1 } { ( 1 - \rho ) \Gamma ( m ) } ( \frac { ( ( x + \gamma _ { t h } ) y } { \rho } ) ^ { \frac { m - 1 } { 2 } } e ^ { - ( \frac { x + \gamma _ { t h } + y } { 1 - \rho } ) \frac { m } { \overline { \gamma } _ { 1 } } } I _ { m - 1 } \left(\frac{\left.\left.2 m \sqrt{\rho\left(x+\gamma_{t h}\right) y}\right)\right] d x d y}{\bar{\gamma}_{1}(1-\rho)}\right.\right.
\end{aligned}
$$

Further, (10) can be represented as

$$
\mathcal{P}_{o}\left(\gamma_{t h}\right)=1-P_{1}+P_{2}
$$

where

$$
\begin{aligned}
P_{1} & =\left(\frac{m}{\bar{\gamma}_{1}}\right)^{m+1} \frac{1}{(1-\rho) \Gamma(m)} \int_{0}^{\infty} \int_{0}^{\infty}\left(\frac{\left(\left(x+\gamma_{t h}\right) y\right.}{\rho}\right)^{\frac{m-1}{2}} e^{-\left(\frac{x+\gamma_{t h}+y}{1-\rho}\right) \frac{m}{\gamma_{1}}} \\
& \times I_{m-1}\left(\frac{2 m \sqrt{\rho\left(x+\gamma_{t h}\right) y}}{\bar{\gamma}_{1}(1-\rho)}\right) d x d y \\
P_{2} & =\left(\frac{m}{\bar{\gamma}_{1}}\right)^{m+1} \frac{A}{(1-\rho) \Gamma(m)} \int_{0}^{\infty} \int_{0}^{\infty}\left(\frac{\left(\left(x+\gamma_{t h}\right) y\right.}{\rho}\right)^{\frac{m-1}{2}} e^{-\left(\frac{x+\gamma_{t h}+y}{1-\rho}\right) \frac{m}{\bar{\gamma}_{1}}} \\
& \times I_{m-1}\left(\frac{2 m \sqrt{\rho\left(x+\gamma_{t h}\right) y}}{\bar{\gamma}_{1}(1-\rho)}\right) \mathrm{G}_{i+1,3 i+1}^{3 i, 1}\left[\left.\frac{B \gamma_{t h}}{\Omega_{i}} \frac{y}{x}\right|_{\mu_{2}, 0} ^{1, \mu_{1}}\right] d x d y .
\end{aligned}
$$

The infinite series representation of the $v$ th order modified Bessels function of first kind can be expressed as

$$
I_{v}(x)=\sum_{j=0}^{\infty} \frac{\left(\frac{1}{2}\right)^{v+2 j}}{j ! \Gamma(v+j+1)} x^{v+2 j} .
$$

Using (14), $P_{1}$ can be written as

$$
\begin{aligned}
P_{1} & =\left(\frac{m}{\bar{\gamma}_{1}}\right)^{m+1} \frac{1}{\rho^{\frac{(m-1)}{2}}(1-\rho) \Gamma(m)} \sum_{j=0}^{\infty} \frac{\left(\frac{1}{2}\right)^{m-1+2 j}}{j ! \Gamma(m+j)}\left(\frac{2 m \sqrt{\rho}}{\bar{\gamma}_{1}(1-\rho)}\right)^{m-1+2 j} \\
& \times \int_{0}^{\infty} \int_{0}^{\infty}\left(\left(x+\gamma_{t h}\right) y\right)^{m-1+j} e^{-\left(x+\gamma_{t h}+y\right) \frac{m}{(1-\rho) \bar{\gamma}_{1}}} d x d y
\end{aligned}
$$

This can be solved as

$$
P_{1}=C_{1} C_{2}^{-2(m+j)} \Gamma(m+j) \Gamma\left(m+j, C_{2} G_{t h}\right)
$$


where $C_{1}=\left(\frac{m}{\bar{\gamma}_{1}}\right)^{m+1} \frac{1}{(1-\rho) \Gamma(m)} \sum_{j=0}^{\infty} \frac{1}{j ! \Gamma(m+j)} C_{2}^{m-1+2 j} \rho^{j}$ and $C_{2}=\frac{m}{(1-\rho) \bar{\gamma}_{1}}$. Substituting the infinite series representation of the $I_{v}(x)$ from (14), $P_{2}$ can be written as

$$
\begin{aligned}
P_{2} & =\left(\frac{m}{\bar{\gamma}_{1}}\right)^{m+1} \frac{A}{\rho^{\frac{(m-1)}{2}}(1-\rho) \Gamma(m)} \sum_{j=0}^{\infty} \frac{\left(\frac{1}{2}\right)^{m-1+2 j}}{j ! \Gamma(m+j)} \\
& \times \int_{0}^{\infty} \int_{0}^{\infty}\left(\left(x+\gamma_{t h}\right) y\right)^{m-1+j} e^{-C_{2}\left(x+\gamma_{t h}+y\right)} \mathrm{G}_{i+1,3 i+1}^{3 i, 1}\left[\left.\frac{B \gamma_{t h}}{\Omega_{i}} \frac{y}{x}\right|_{\mu_{2}, 0} ^{1, \mu_{1}}\right] d x d y .
\end{aligned}
$$

Further, (17) can be modified as

$$
\begin{aligned}
P_{2} & =A C_{1} C_{2}^{-(m+j)} e^{-C_{2} \gamma_{t h}} \sum_{z_{1}=0}^{m-1+j}\left(\begin{array}{c}
m-1+j \\
z_{1}
\end{array}\right) \gamma_{t h}^{m-1+j-z_{1}} \\
& \times \int_{0}^{\infty} x^{z_{1}} e^{-C_{2} x} \mathrm{G}_{3 i+1, i+2}^{2,3 i}\left[\left.\frac{\Omega_{i} C_{2}}{B \gamma_{t h}} x\right|_{(m+j), 0,\left(1-\mu_{1}\right)} ^{\left(1-\mu_{2}\right), 1}\right] d x .
\end{aligned}
$$

Solving the required integral with the help of $[33,(7.813)], P_{2}$ can be derived as

$$
P_{2}=A C_{1} \sum_{z_{1}=0}^{m-1+j}\left(\begin{array}{c}
m-1+j \\
z_{1}
\end{array}\right) C_{2}^{-\left(m+j+z_{1}+1\right)} \gamma_{t h}^{m-1+j-z_{1}} e^{-C_{2} \gamma_{t h}} \mathrm{G}_{i+2,3 i+2}^{3 i+1,2}\left[\left.\frac{B \gamma_{t h}}{\Omega_{i}}\right|_{1+z_{1}, \mu_{2}, 0} ^{1-(m+j), 1, \mu_{1}}\right]
$$

Thus, from (11), outage probability can be expressed as

$$
\begin{aligned}
\mathcal{P}_{o}\left(\gamma_{t h}\right) & =1-C_{1}\left\{C_{2}^{-2(m+j)} \Gamma(m+j) \Gamma\left(m+j, C_{2} \gamma_{t h}\right)-A \sum_{z_{1}=0}^{m-1+j}\left(\begin{array}{c}
m-1+j \\
z_{1}
\end{array}\right) C_{2}^{-\left(m+j+z_{1}+1\right)}\right. \\
& \left.\times \gamma_{t h}^{m-1+j-z_{1}} e^{-C_{2} \gamma_{t h}} \mathrm{G}_{i+2,3 i+2}^{3 i+1,2}\left[\left.\frac{B \gamma_{t h}}{\Omega_{i}}\right|_{1+z_{1}, \mu_{2}, 0} ^{1-(m+j), 1, \mu_{1}}\right]\right\} .
\end{aligned}
$$

If only a single term of summation is considered $(j=0)$, outage probability is approximated as

$$
\begin{aligned}
\mathcal{P}_{o}\left(\gamma_{t h}\right) & =1-\frac{1}{(\Gamma(m))^{2}}\left(\frac{m}{\bar{\gamma}_{1}}\right)^{2 m}\left(\frac{1}{1-\rho}\right)^{m}\left\{C_{2}^{-2 m} \Gamma(m) \Gamma\left(m, C_{2} \gamma_{t h}\right)\right. \\
& \left.-A \sum_{z_{1}=0}^{m-1}\left(\begin{array}{c}
m-1 \\
z_{1}
\end{array}\right) C_{2}^{-\left(m+z_{1}+1\right)} \gamma_{t h}^{m-1-z_{1}} e^{-C_{2} \gamma_{t h}} \mathrm{G}_{i+2,3 i+2}^{3 i+1,2}\left[\left.\frac{B \gamma_{t h}}{\Omega_{i}}\right|_{1+z_{1}, \mu_{2}, 0} ^{1-m, 1, \mu_{1}}\right]\right\} .
\end{aligned}
$$

Considering two extreme cases, when the CSI is uncorrelated $(\rho=0)$ and when the CSI is perfectly correlated $(\rho=1)$.

A. Uncorrelated CSI $(\rho=0)$ :

For uncorrelated case, substituting $(\rho=0)$ in $(21)$, outage probability can be expressed as

$$
\begin{aligned}
\mathcal{P}_{o}\left(\gamma_{t h}\right) & =1-\frac{1}{\Gamma(m)} \Gamma\left(m, \frac{m}{\bar{\gamma}_{1}} \gamma_{t h}\right)-\frac{A}{(\Gamma(m))^{2}}\left(\frac{m}{\bar{\gamma}_{1}}\right)^{2 m} \sum_{z_{1}=0}^{m-1}\left(\begin{array}{c}
m-1 \\
z_{1}
\end{array}\right)\left(\frac{m}{\bar{\gamma}_{1}}\right)^{-\left(m+z_{1}+1\right)} \\
& \times \gamma_{t h}^{m-1-z_{1}} e^{-\frac{m}{\bar{\gamma}_{1}} \gamma_{t h}} \mathrm{G}_{i+2,3 i+2}^{3 i+1,2}\left[\left.\frac{B \gamma_{t h}}{\Omega_{i}}\right|_{1+z_{1}, \mu_{2}, 0} ^{1-m, 1, \mu_{1}}\right] .
\end{aligned}
$$

\section{B. Perfectly Correlated CSI $(\rho=1)$ :}

For perfect CSI, $\rho=1$, e2e SNR can be written as $\gamma=\frac{\gamma_{1} \gamma_{2}}{\gamma_{1}+\gamma_{2}}$ which is upper bounded as $\gamma=\min \left(\gamma_{1}, \gamma_{2}\right)$. Thus, outage probability can be derived as

$$
\begin{aligned}
\mathcal{P}_{o}\left(\gamma_{t h}\right) & =1-\bar{F}_{s r}\left(\gamma_{t h}\right) \bar{F}_{r d}\left(\gamma_{t h}\right) \\
& =1-\frac{1}{\Gamma(m)} \Gamma\left(m, \frac{m \gamma_{t h}}{\bar{\gamma}_{1}}\right)\left\{1-A G_{i+1,3 i+1}^{3 i, 1}\left[\left.\frac{B}{\Omega_{i}} \gamma_{t h}\right|_{\mu_{2}, 0} ^{1, \mu_{1}}\right]\right\} .
\end{aligned}
$$




\section{Asymptotic Outage Probability}

To get more insights from the outage probability, it is simplified under certain conditions by neglecting the higher order terms of the series expansion of the modified Bessel function of first kind. This can be done by considering only a single term of the summation $(\mathrm{j}=0)$, which is shown in (21). However, this is valid only when the argument of $I_{0}(\cdot)$ tends to zero at high SNRs or the $\rho$ is small enough. Accuracy of approximation further depends on the FSO channel condition. As Gamma-Gamma distribution is considered for FSO channel, Meijer-G function plays an important role in outage probability expression. Approximation (21) is not accurate when atmospheric turbulence is strong or the argument of Meijer-G Function is large (average SNR of FSO channel is low). Thus, more number of summation terms are required for better accuracy [8]. Now considering the high SNR approximation, the Meijer-G function presents in (20) can be expressed in basic elementary function terms as [34, (07.34.06.0001.01)]

$$
\mathrm{G}_{i+2,3 i+2}^{3 i+1,2}\left[\left.\frac{B \gamma_{t h}}{\Omega_{i}}\right|_{\mu_{4}} ^{\mu_{3}}\right]=\sum_{k=1}^{3 i+1}\left(\frac{B \gamma_{t h}}{\Omega_{i}}\right)^{\mu_{4}, k} \frac{\prod_{\substack{l=1 \\ l \neq k}}^{3 i+1} \Gamma\left(\mu_{4, l}-\mu_{4, k}\right) \prod_{l=1}^{2} \Gamma\left(1-\mu_{3, l}+\mu_{4, k}\right)}{\prod_{l=3}^{i+2} \Gamma\left(\mu_{3, l}-\mu_{4, k}\right) \prod_{3 i+2}^{3 i+2} \Gamma\left(1-\mu_{4, l}+\mu_{4, k}\right)},
$$

where $\mu_{3}=\left[1-(m+j), 1, \mu_{1}\right]$ and $\mu_{4}=\left[1+z_{1}, \mu_{2}, 0\right]$. From (24), the dominant term in the asymptotic expression of meijer-G function is $\min \left(\frac{\xi^{2}}{i}, \frac{\alpha}{i}, \frac{\beta}{i}\right)$. Substituting (24) in (20), asymptotic outage probability expression at high SNR can be given by

$$
\begin{aligned}
\mathcal{P}_{o}^{a s y m}\left(\gamma_{t h}\right) & =1-C_{1}\left\{C_{2}^{-2(m+j)} \Gamma(m+j) \Gamma\left(m+j, C_{2} \gamma_{t h}\right)-A \sum_{z_{1}=0}^{m-1+j}\left(\begin{array}{c}
m-1+j \\
z_{1}
\end{array}\right) C_{2}^{-\left(m+j+z_{1}+1\right)} \gamma_{t h}^{m-1+j-z_{1}}\right. \\
& \left.\times e^{-C_{2} \gamma_{t h}} \sum_{k=1}^{3 i+1}\left(\frac{B \gamma_{t h}}{\Omega_{i}}\right)^{\mu_{4}, k} \frac{\prod_{\substack{l=1 \\
l \neq k}}^{3 i+1} \Gamma\left(\mu_{4, l}-\mu_{4, k}\right) \prod_{l=1}^{2} \Gamma\left(1-\mu_{3, l}+\mu_{4, k}\right)}{\prod_{l=3}^{i+2} \Gamma\left(\mu_{3, l}-\mu_{4, k}\right) \prod_{3 i+2}^{3 i+2} \Gamma\left(1-\mu_{4, l}+\mu_{4, k}\right)}\right\} .
\end{aligned}
$$

As a mixed RF/FSO system is considered, the diversity order is given by $\min \left(m, \frac{\xi^{2}}{i}, \frac{\alpha}{i}, \frac{\beta}{i}\right)$, where $m$ is the fading coefficient of the RF link and $\frac{\xi^{2}}{i}, \frac{\alpha}{i}, \frac{\beta}{i}$ are the dominant terms of the FSO link.

\section{ERGODIC CAPACITY}

In this Section, ergodic capacity of the considered RF/FSO system is derived. Generalized ergodic capacity expression (in bits $/ \mathrm{sec} / \mathrm{Hz}$ ) can be computed as

$$
\mathcal{C}_{e}=\int_{0}^{\infty} \log _{2}(1+\gamma) f_{\gamma}(\gamma) d \gamma
$$

where $f_{\gamma}(\gamma)$ represents the PDF of the e2e SNR. Ergodic capacity given in (26), can be modified in CDF form as

$$
\mathcal{C}_{e}=\frac{1}{\ln (2)} \int_{0}^{\infty} \frac{1}{1+\gamma}\left(1-\mathcal{P}_{o}(\gamma)\right) d \gamma
$$

where $\mathcal{P}_{o}(\gamma)$ represents the outage probability or the CDF of the e2e SNR. Representing the identity $\left(1+l_{1} \gamma\right)^{-l_{2}}=$ $\frac{1}{\Gamma\left(l_{2}\right)} \mathrm{G}_{1,1}^{1,1}\left[\left.l_{1} \gamma\right|_{0} ^{1-l_{2}}\right],(27)$ can be written as

$$
\mathcal{C}_{e}=\frac{1}{\ln (2)} \int_{0}^{\infty} \mathrm{G}_{1,1}^{1,1}\left[\left.\gamma\right|_{0} ^{0}\right]\left(1-\mathcal{P}_{o}(\gamma)\right) d \gamma
$$

Substituting (20) in (28), we obtain

$$
\begin{aligned}
\mathcal{C}_{e} & =\frac{1}{\ln (2)} C_{1}\left\{C_{2}^{-2(m+j)} \Gamma(m+j) \int_{0}^{\infty} \mathrm{G}_{1,1}^{1,1}\left[\left.\gamma\right|_{0} ^{0}\right] \Gamma\left(m+j, C_{2} \gamma\right) d \gamma-A \sum_{z_{1}=0}^{m-1+j}\left(\begin{array}{c}
m-1+j \\
z_{1}
\end{array}\right)\right. \\
& \left.\times C_{2}^{-\left(m+j+z_{1}+1\right)} \int_{0}^{\infty} \gamma_{t h}^{m-1+j-z_{1}} e^{-C_{2} \gamma_{t h}} \mathrm{G}_{1,1}^{1,1}\left[\left.\gamma\right|_{0} ^{0}\right] \mathrm{G}_{i+2,3 i+2}^{3 i+1,2}\left[\left.\frac{B \gamma_{t h}}{\Omega_{i}}\right|_{1+z_{1}, \mu_{2}, 0} ^{1-(m+j), 1, \mu_{1}}\right] d \gamma\right\} .
\end{aligned}
$$


Representing the upper incomplete Gamma function in its series form and exponential function in terms of Meijer-G function, ergodic capacity for the considered system can be expressed in terms of extended generalized bivariate Meijer-G function (EGBMGF) as ${ }^{2}$

$$
\begin{aligned}
\mathcal{C}_{e} & =\frac{1}{\ln (2)} C_{1}\left\{C_{2}^{-2(m+j)} \Gamma(m+j) \sum_{j_{1}=0}^{m+j-1} \frac{C_{2}^{j_{1}}}{j_{1} !} \mathrm{G}_{2,1}^{1,2}\left[\left.\frac{1}{C_{2}}\right|_{0} ^{-j_{1}, 0}\right]\right. \\
& \left.-A \sum_{z_{1}=0}^{m-1+j}\left(\begin{array}{c}
m-1+j \\
z_{1}
\end{array}\right) C_{2}^{-\left(m+j+z_{1}+1\right)}\left(\frac{B}{\Omega_{i}}\right)^{-C_{3}} \mathrm{G}_{3 i+2, i+2,: 0,1: 1,1}^{2,3 i+1: 1,0: 1,1}\left[\frac{C_{2} \Omega_{i}}{B},\left.\left.\left.\frac{\Omega_{i}}{B}\right|_{-C_{3}+m+j,-C_{3}, 1-C_{3}-\mu_{1}}\right|_{0} ^{-\left(C_{3}+z_{1}\right), 1-C_{3}-\mu_{2}, 1-C_{3}}\right|_{0} ^{-}\right]\right\},
\end{aligned}
$$

where $C_{3}=\left(m+j-z_{1}\right)$.

\section{ASER ANALYSIS}

Based on the CDF of the e2e instantaneous SNR, for any digital modulation technique, generalized ASER expression is given as

$$
\mathcal{P}_{e}=-\int_{0}^{\infty} \mathcal{P}_{s}^{\prime}(e \mid \gamma) \mathcal{P}_{o}(\gamma) d \gamma
$$

where $\mathcal{P}_{s}^{\prime}(e \mid \gamma)$ represents the first order derivative of the conditional SEP $\left(\mathcal{P}_{s}(e \mid \gamma)\right)$ for the received SNR.

\section{A. Hexagonal QAM}

For M-ary HQAM scheme, conditional SEP expression for AWGN channel can be given as

$$
\mathcal{P}_{s}^{H}(e \mid \gamma)=Q(\sqrt{K \gamma})\left[\tau-2 \tau_{c} Q\left(\sqrt{\frac{K \gamma}{3}}\right)\right]+\frac{2}{3} \tau_{c} Q^{2}\left(\sqrt{\frac{2 K \gamma}{3}}\right),
$$

where $K, \tau$, and $\tau_{c}$ are constants, and their different values are used to select various HQAM constellations.

TABLE I: Different parameters for the various regular and irregular HQAM constellations over the AWGN channel.

\begin{tabular}{|c|c|c|c|c|c|c|}
\hline & \multicolumn{3}{|c|}{ Regular HQAM } & \multicolumn{3}{c|}{ Irregular HQAM (optimum) } \\
\hline $\mathrm{M}$ & $\mathrm{K}$ & $\tau$ & $\tau_{c}$ & $\mathrm{~K}$ & $\tau$ & $\tau_{c}$ \\
\hline 4 & 1 & $\frac{5}{2}$ & $\frac{3}{2}$ & 1 & $\frac{5}{2}$ & $\frac{3}{2}$ \\
\hline 8 & $\frac{2}{6}$ & $\frac{7}{2}$ & $\frac{21}{8}$ & $\frac{32}{69}$ & $\frac{7}{2}$ & $\frac{21}{8}$ \\
\hline 16 & $\frac{2}{9}$ & $\frac{33}{8}$ & $\frac{27}{8}$ & $\frac{8}{35}$ & $\frac{33}{8}$ & $\frac{27}{8}$ \\
\hline 32 & $\frac{8}{71}$ & $\frac{75}{16}$ & $\frac{33}{8}$ & $\frac{512}{4503}$ & $\frac{75}{16}$ & $\frac{33}{8}$ \\
\hline 64 & $\frac{2}{37}$ & $\frac{161}{32}$ & $\frac{147}{32}$ & $\frac{8}{141}$ & $\frac{163}{32}$ & $\frac{75}{16}$ \\
\hline 128 & $\frac{2}{72}$ & $\frac{339}{64}$ & $\frac{159}{32}$ & $\frac{2}{70.56}$ & $\frac{343}{64}$ & $\frac{81}{16}$ \\
\hline 256 & $\frac{2}{149}$ & $\frac{705}{128}$ & $\frac{675}{128}$ & $\frac{2}{141}$ & $\frac{711}{128}$ & $\frac{171}{32}$ \\
\hline 512 & $\frac{2}{289.06}$ & $\frac{2895}{512}$ & $\frac{5619}{1024}$ & $\frac{200}{28217}$ & $\frac{2911}{512}$ & $\frac{5667}{1024}$ \\
\hline 1024 & $\frac{2}{597}$ & $\frac{2945}{512}$ & $\frac{2883}{512}$ & $\frac{100}{28227}$ & $\frac{2955}{512}$ & $\frac{1449}{256}$ \\
\hline
\end{tabular}

For an $M$-ary HQAM constellation

$$
\tau=\frac{1}{M} \sum_{i=0}^{M-1} \tau(i), \tau_{c}=\frac{1}{M} \sum_{i=0}^{M-1} \tau_{c}(i), K=\frac{N_{0}}{E_{s}}\left(\frac{d}{2 \sigma}\right)^{2}=\frac{N_{0}}{4 \sigma^{2}} \times \frac{d^{2}}{E_{s}},
$$

where $\tau(i)$ and $\tau_{c}(i)$ represent the nearest neighborhoods (NNs) and couple of NNs, respectively for symbol $s(i)$. Further, $d$ is the minimum distance between the constellation points and $\sigma$ is the standard deviation of the AWGN. For a well connected HQAM, $\tau_{c}=3 T / M$, where T represents the number of equilateral triangles with sides of length $d$, and $\tau=2\left[\frac{\tau_{c}}{3}+1-\frac{1}{M}\right]$ [23]. For a regular HQAM structure with constellation of even power of $2, K=\frac{24}{7 M-4}, \tau=2\left(3-\frac{4}{\sqrt{M}}+\frac{1}{M}\right)$ and $\tau_{c}=6\left(1-\frac{1}{\sqrt{M}}\right)^{2}$. However, for the constellations with arbitrary power of 2, no such pattern can be found in the literature for different values of $K, \tau$ and $\tau_{c}$ for both regular and irregular HQAM schemes. 


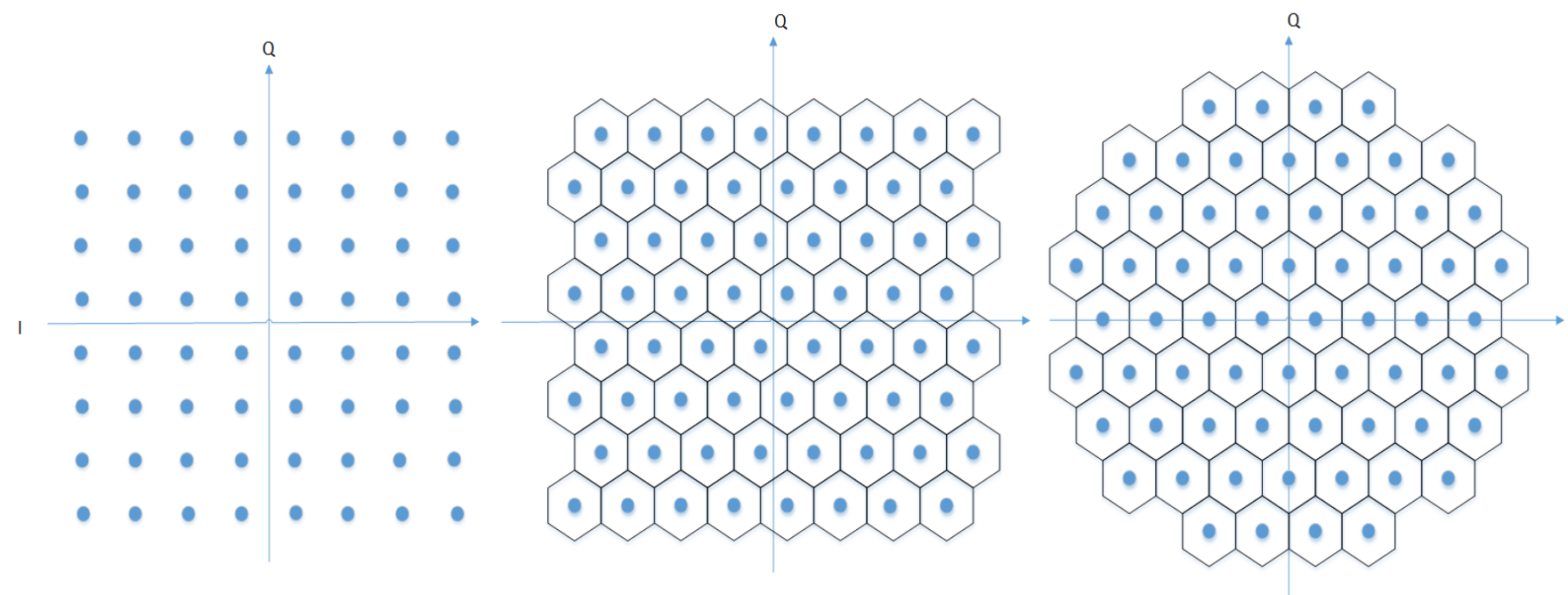

Fig. 1: Generation of an irregular HQAM constellation (M=64)

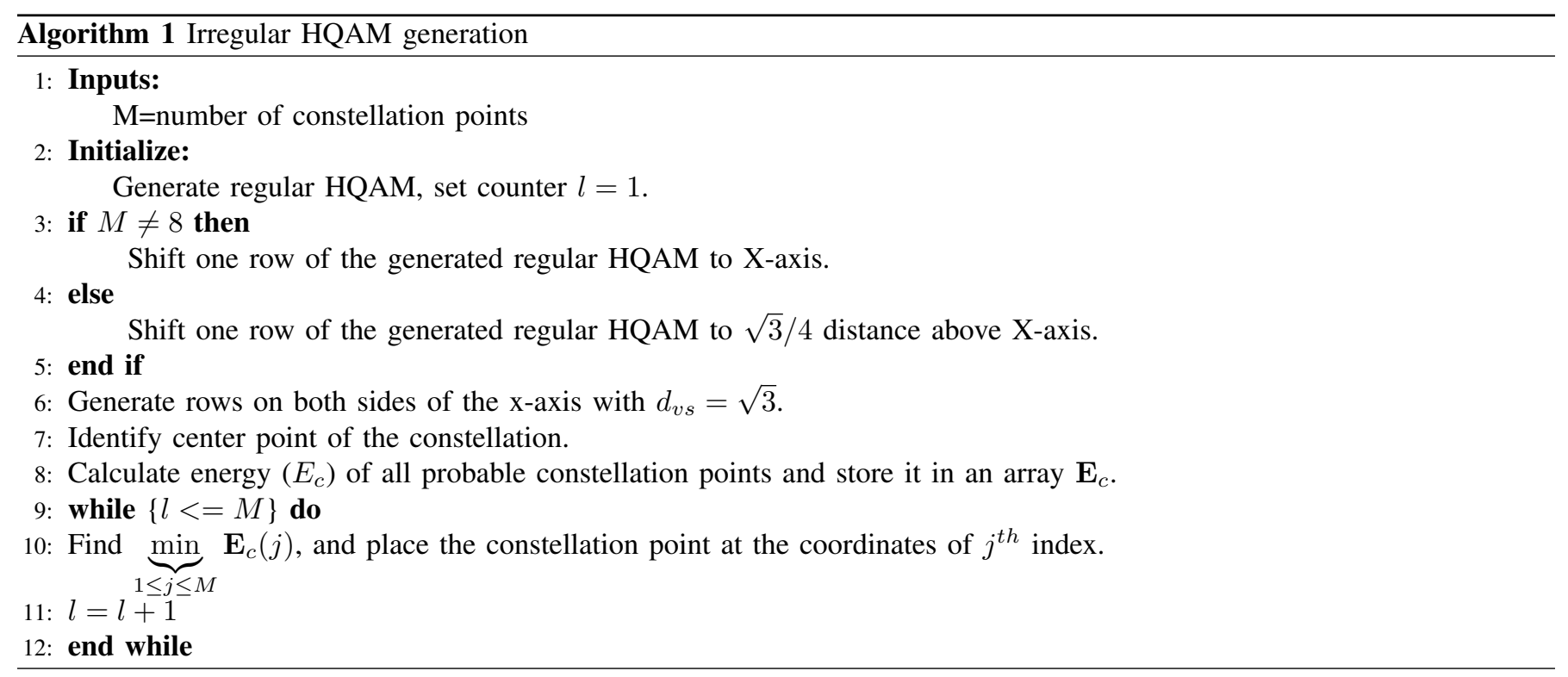

Irregular HQAM Constellations: Algorithm 1 illustrates generation of the irregular HQAM (optimum) constellation (Fig. 1). Here, $d_{v s}$ represents the vertical spacing, $E_{c}$ represents the energy of a constellation point, and $\mathbf{E}_{c}$ represents the array of constellation points energies.

Various irregular HQAM constellations are shown in Fig. 2, and different values of $K, \tau$, and $\tau_{c}$ for various regular and irregular HQAM constellations are calculated manually which are summarized in Table I. A detailed study on various HQAM or TQAM constellations ${ }^{3}$ can be seen in [1], [23], [26]-[28], [36]. Further, solving the equations given for $K, \tau$, and $\tau_{c}$, generalized approximate values of $K, \tau$, and $\tau_{c}$, for a well connected $M$-ary irregular HQAM can be given as

$$
\begin{aligned}
K & =\frac{7}{2 M-1}, \\
\tau & =6.07-6.733 M^{-0.456}, \\
\tau_{c} & =3\left[\frac{\tau}{2}-1+\frac{1}{M}\right] .
\end{aligned}
$$

Substituting the Gaussian Q-function $Q(z)=\frac{1}{2}\left[1-\operatorname{erf}\left(\frac{z}{\sqrt{2}}\right)\right]$ in (32) and using [37, (7.1.21)], first order derivative of (32) can be expressed as [2], [3]

\footnotetext{
${ }^{2}$ Mathematica implementation of extended generalized bivariate Meijer-G function is given in [35].

${ }^{3}$ Placement of the constellation points on the 2D plane is similar for both the HQAM and TQAM schemes. If the constellation is termed as HQAM, the constellation points are placed at the center of a hexagon and separated with the neighboring point hexagon with $2 d$ distance. However, if the constellation is termed as TQAM, each constellation point is placed on the vertex of well connected equilateral triangles of side-length $2 d$. However, the decision boundaries will be in hexagon shape.
} 


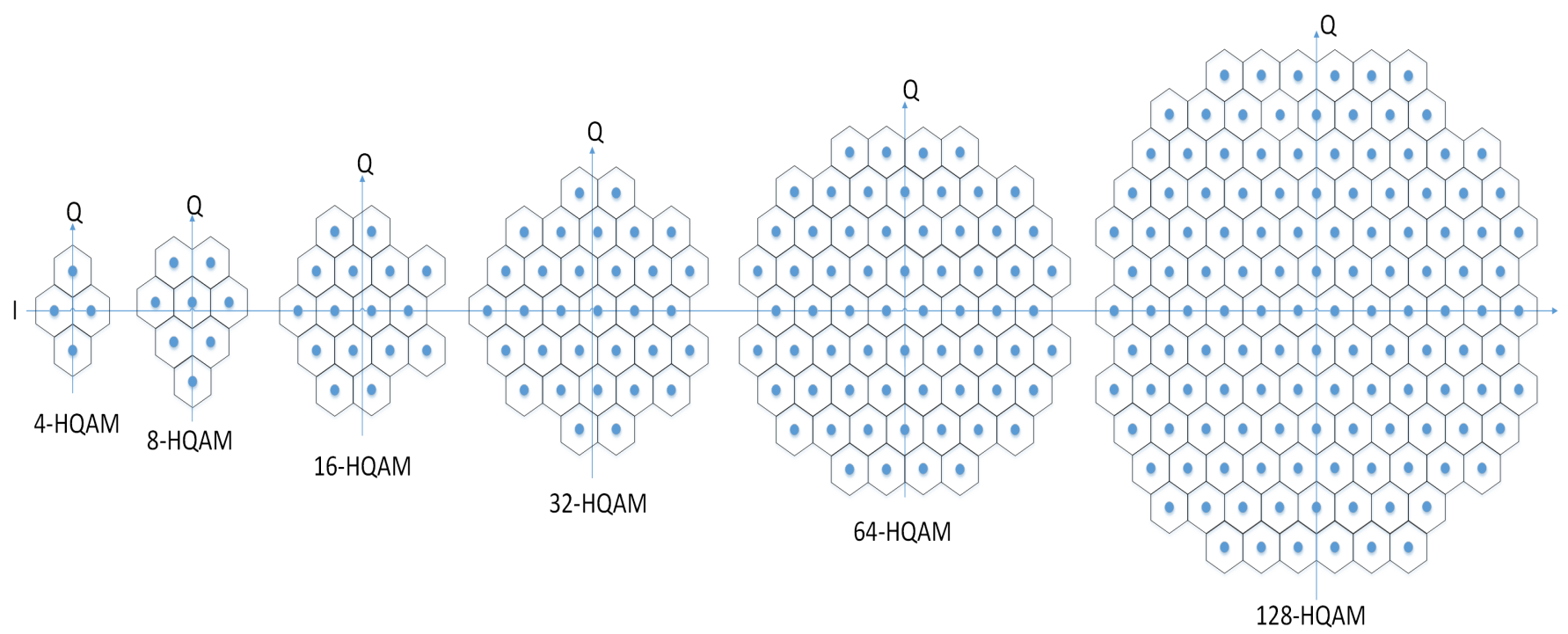

(a)

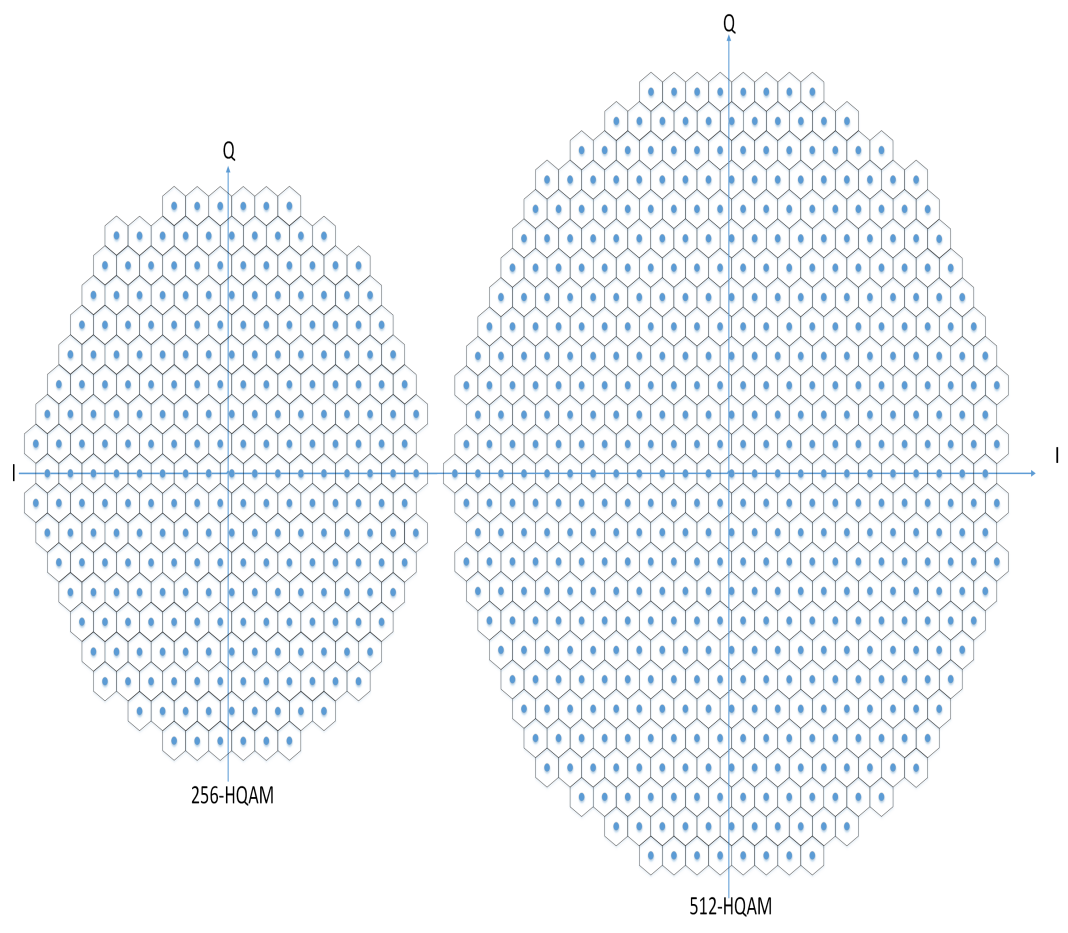

(b)

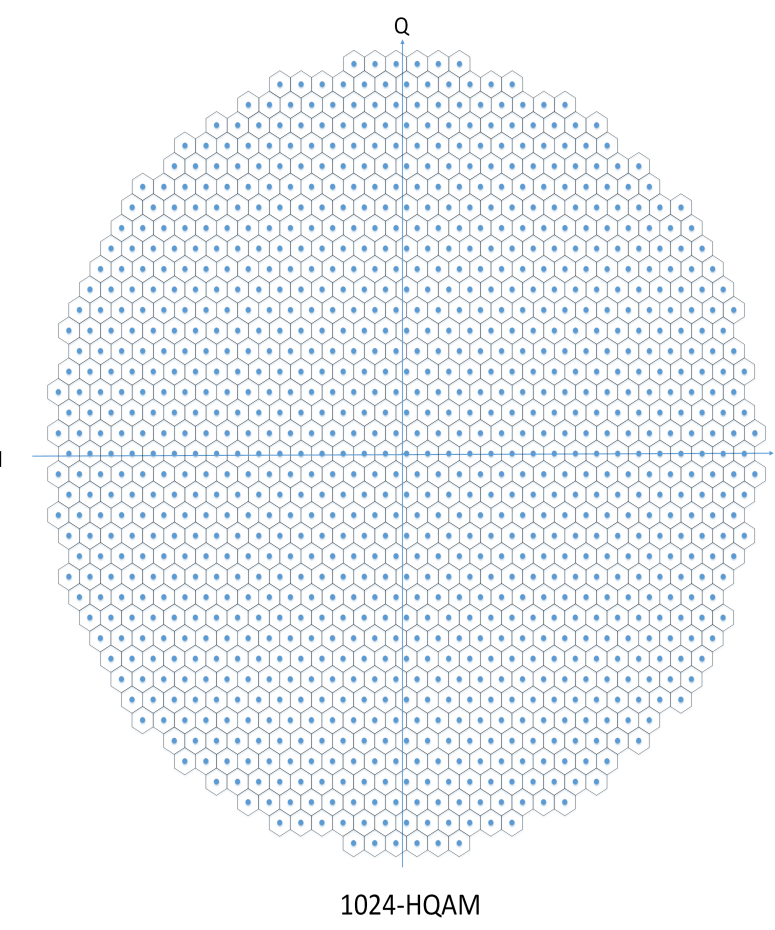

(c)

Fig. 2: Various irregular HQAM (optimum) constellations.

$$
\begin{aligned}
\mathcal{P}_{s}^{\prime H}(e \mid \gamma) & =\gamma^{-1 / 2}\left[\frac{1}{2} \sqrt{\frac{K}{2 \pi}}\left(\tau_{c}-\tau\right) e^{-\frac{K \gamma}{2}}-\frac{\tau_{c}}{3} \sqrt{\frac{K}{3 \pi}} e^{-\frac{K \gamma}{3}}+\frac{\tau_{c}}{2} \sqrt{\frac{K}{6 \pi}} e^{-\frac{K \gamma}{6}}\right] \\
& +\frac{2 \tau_{c} K}{9 \pi} e^{-\frac{2 K}{3} \gamma}{ }_{1} F_{1}\left(1 ; \frac{3}{2} ; \frac{K}{3} \gamma\right)-\frac{\tau_{c} K e^{-\frac{2 K}{3} \gamma}}{2 \sqrt{3} \pi}\left[{ }_{1} F_{1}\left(1 ; \frac{3}{2} ; \frac{K}{2} \gamma\right)+{ }_{1} F_{1}\left(1 ; \frac{3}{2} ; \frac{K}{6} \gamma\right)\right] .
\end{aligned}
$$

Substituting $\mathcal{P}_{s}^{\prime H}(e \mid \gamma)$ and $\mathcal{P}_{o}(\gamma)$, respectively from (35) and (20) in (31), and after solving the required integrals with the help of $[33,(3.351 .3),(6.455 .1),(7.813 .1)]$, the generalized expression for ASER for both regular and irregular HQAM scheme is given as 
TABLE II: Various functions used in analytical expressions with their values.

\begin{tabular}{|l|l|}
\hline Function & Value \\
\hline \hline$C_{3}$ & $\sum_{z=0}^{\infty} \frac{(1)_{z}}{z !(1.5)_{z}}\left[\frac{2 \tau_{c} K}{9 \pi}\left(\frac{K}{3}\right)^{2}-\left(\frac{\tau_{c} K}{2 \sqrt{3} \pi}\left(\left(\frac{K}{2}\right)^{z}+\left(\frac{K}{6}\right)^{2}\right)\right)\right]$ \\
\hline $\mathbb{F}(\theta, \phi)$ & $\Gamma(\theta) \phi^{-\theta}$ \\
\hline $\mathbb{G}(\theta, \phi)$ & $\frac{C_{2}^{m+j} \Gamma(m+j+\theta)}{\theta\left(C_{2}+\phi\right)^{m+j+\theta}}{ }_{2} F_{1}\left[1, m+j+\theta ; \theta+1 ; \frac{\phi}{\phi+C_{2}}\right]$ \\
\hline $\mathbb{H}(\theta, \phi)$ & $\left(C_{2}+\phi\right)^{-\left(m-1+j-z_{1}+\theta\right)} \mathrm{G}_{i+3,3 i+2}^{3 i+1,3}\left[\left.\frac{B}{\Omega_{i}\left(C_{2}+\phi\right)}\right|_{1+z_{1}, \mu_{2}, 0} ^{-(m-1+j-z+\theta-1), 1-(m+j), 1, \mu_{1}}\right]$ \\
\hline$C_{4}$ & $\frac{a_{0} b_{0} p_{0} q_{0}}{\pi} \sum_{z=0}^{\infty} \frac{(1)_{z}}{z !(1.5)_{z}}\left[\left(\frac{a_{0}^{2}}{2}\right)^{z}+\left(\frac{b_{0}^{2}}{2}\right)^{z}\right]$ \\
\hline
\end{tabular}

$$
\begin{aligned}
\mathcal{P}_{e}^{H} & =\left[\frac{1}{2} \sqrt{\frac{K}{2 \pi}}\left(\tau_{c}-\tau\right) \mathbb{F}\left(\frac{1}{2}, \frac{K}{2}\right)-\frac{\tau_{c}}{3} \mathbb{F}\left(\frac{1}{2}, \frac{K}{3}\right)+\frac{\tau_{c}}{2} \sqrt{\frac{K}{6 \pi}} \mathbb{F}\left(\frac{1}{2}, \frac{K}{6}\right)+C_{3} \mathbb{F}\left(z+1, \frac{2 K}{3}\right)\right] \\
& -C_{1} C_{2}^{-2(m+j)} \Gamma(m+j)\left[\frac{1}{2} \sqrt{\frac{K}{2 \pi}}\left(\tau_{c}-\tau\right) \mathbb{G}\left(\frac{1}{2}, \frac{K}{2}\right)-\frac{\tau_{c}}{3} \mathbb{G}\left(\frac{1}{2}, \frac{K}{3}\right)+\frac{\tau_{c}}{2} \sqrt{\frac{K}{6 \pi}} \mathbb{G}\left(\frac{1}{2}, \frac{K}{6}\right)+C_{3} \mathbb{G}\left(z+1, \frac{2 K}{3}\right)\right] \\
& +A C_{1} \sum_{z_{1}=0}^{m-1+j}\left(\begin{array}{c}
m-1+j \\
z_{1}
\end{array}\right) C_{2}^{-\left(m+j+z_{1}+1\right)}\left[\frac{1}{2} \sqrt{\frac{K}{2 \pi}}\left(\tau_{c}-\tau\right) \mathbb{H}\left(\frac{1}{2}, \frac{K}{2}\right)-\frac{\tau_{c}}{3} \mathbb{H}\left(\frac{1}{2}, \frac{K}{3}\right)\right. \\
& \left.+\frac{\tau_{c}}{2} \sqrt{\frac{K}{6 \pi}} \mathbb{H}\left(\frac{1}{2}, \frac{K}{6}\right)+C_{3} \mathbb{H}\left(z+1, \frac{2 K}{3}\right)\right],
\end{aligned}
$$

where $C_{3}, \mathbb{F}(\theta, \phi), \mathbb{G}(\theta, \phi)$, and $\mathbb{H}(\theta, \phi)$ are given in Table II.

\section{B. Rectangular $Q A M$}

For $M_{I} \times M_{Q}$-ary QAM scheme, generalized conditional SEP expression $\left(\mathcal{P}_{s}^{R}(e \mid \gamma)\right)$ and its first order derivative $\left(\mathcal{P}_{s}^{\prime R}(e \mid \gamma)\right)$ for AWGN channel are given in [4, (41)] and [4, (42)], respectively, where $p_{0}=1-\left(1 / M_{I}\right), q_{0}=1-\left(1 / M_{Q}\right), a_{0}=$ $\sqrt{6 /\left(\left(M_{I}^{2}-1\right)+\left(M_{Q}^{2}-1\right) \lambda^{2}\right)}, b_{0}=\lambda a_{0}$, and $\lambda=d_{Q} / d_{I}$ represents the ratio of quadrature and in-phase decision distances. Also, $M_{I}$ and $M_{Q}$ represent respectively the in-phase and quadrature phase constellation points.

Substituting $\mathcal{P}_{s}^{\prime R}(e \mid \gamma)$ and $\mathcal{P}_{o}(\gamma)$ from [4, (42)] and (20), respectively in (31), and after some mathematical computations, analytical ASER expression for the general order RQAM can be given as

$$
\begin{aligned}
\mathcal{P}_{e}^{R} & =\left[\frac{a_{0} p_{0}\left(1-q_{0}\right)}{\sqrt{2 \pi}} \mathbb{F}\left(\frac{1}{2}, \frac{a_{0}^{2}}{2}\right)+\frac{b_{0}\left(1-p_{0}\right) q_{0}}{\sqrt{2 \pi}} \mathbb{F}\left(\frac{1}{2}, \frac{b_{0}^{2}}{2}\right)+C_{4} \mathbb{F}\left(z+1, \frac{a_{0}^{2}+b_{0}^{2}}{2}\right)\right]-C_{1} C_{2}^{-2(m+j)} \Gamma(m+j) \\
& \times\left[\frac{a_{0} p_{0}\left(1-q_{0}\right)}{\sqrt{2 \pi}} \mathbb{G}\left(\frac{1}{2}, \frac{a_{0}^{2}}{2}\right)+\frac{b_{0}\left(1-p_{0}\right) q_{0}}{\sqrt{2 \pi}} \mathbb{G}\left(\frac{1}{2}, \frac{b_{0}^{2}}{2}\right)+C_{4} \mathbb{G}\left(z+1, \frac{a_{0}^{2}+b_{0}^{2}}{2}\right)\right]+A C_{1} \sum_{z_{1}=0}^{m-1+j}\left(\begin{array}{c}
m-1+j \\
z_{1}
\end{array}\right) \\
& \times C_{2}^{-\left(m+j+z_{1}+1\right)}\left[\frac{a_{0} p_{0}\left(1-q_{0}\right)}{\sqrt{2 \pi}} \mathbb{H}\left(\frac{1}{2}, \frac{a_{0}^{2}}{2}\right)+\frac{b_{0}\left(1-p_{0}\right) q_{0}}{\sqrt{2 \pi}} \mathbb{H}\left(\frac{1}{2}, \frac{b_{0}^{2}}{2}\right)+C_{4} \mathbb{H}\left(z+1, \frac{a_{0}^{2}+b_{0}^{2}}{2}\right)\right] .
\end{aligned}
$$

\section{Cross $Q A M$}

The conditional SEP of general order XQAM constellation in AWGN channel can be given as [38]

$$
\begin{aligned}
\mathcal{P}_{e}^{X}(e \mid \gamma) & =\left[g_{1} Q(\sqrt{2 \chi \gamma})+\frac{4}{M} Q(2 \sqrt{\chi \gamma})-g_{2} Q^{2}(\sqrt{2 \chi \gamma})-\frac{16}{M} \sum_{t=1}^{v-1} Q\left(\sqrt{2 \chi \gamma}, \alpha_{t}^{+}\right)\right. \\
& \left.-\frac{8}{M} \sum_{t=1}^{v-1} Q\left(2 t \sqrt{\chi \gamma}, \beta_{t}^{+}\right)+\frac{8}{M} \sum_{t=2}^{v} Q\left(2 t \sqrt{\chi \gamma}, \beta_{t}^{-}\right)\right]
\end{aligned}
$$

where $\chi=\frac{48}{31 M-32}, g_{1}=4-\frac{6}{\sqrt{2 M}}, g_{2}=4-\frac{12}{\sqrt{2 M}}+\frac{12}{M}$,

$\alpha_{t}^{+}=\arctan \left(\frac{1}{2 t+1}\right)$, for $t=1, \ldots, v-1$

and $\beta_{t}^{ \pm}=\arctan \left(\frac{t}{t \pm 1}\right)$, for $t=1, \ldots, v$.

Further, $v=\frac{1}{8} \sqrt{2 M}$ decides the XQAM constellations, i.e. $v=1$ for $M=32, v=2$ for $M=128$ and so on. Also, $Q(x, \phi)$ is related to the 1D and 2D Gaussian Q-functions as

$$
Q(x, \phi)=\frac{1}{\pi} \int_{0}^{\phi} \exp \left(-\frac{x^{2}}{2 \sin ^{2} \theta}\right) d \theta \quad \text { for } x \geq 0 .
$$


For the ASER analysis, first order derivative is required and hence, taking the first order derivative of (38), we get

$$
\begin{aligned}
\mathcal{P}_{s}^{\prime X}(e \mid \gamma) & =-\left[\frac{\left(g_{1}-g_{2}\right)}{2} \sqrt{\frac{\chi}{\pi}} \frac{e^{-\chi \gamma}}{\sqrt{\gamma}}+\frac{4}{M} \sqrt{\frac{\chi}{2 \pi}} \frac{e^{-2 \chi \gamma}}{\sqrt{\gamma}}+g_{2} \frac{\chi}{\pi} e^{-2 \chi \gamma}{ }_{1} F_{1}\left(1 ; \frac{3}{2} ; \chi \gamma\right)\right. \\
& +\frac{16}{M} \sum_{t=1}^{v-1} \frac{1}{\pi} \int_{0}^{\alpha_{t}^{+}}-\frac{\chi}{\sin ^{2} \theta} \exp \left(\frac{-\chi \gamma}{\sin ^{2} \theta}\right) d \theta+\frac{8}{M} \sum_{t=1}^{v-1} \frac{1}{\pi} \int_{0}^{\beta_{t}^{+}}-\frac{2 \chi}{\sin ^{2} \theta} \exp \left(\frac{-2 \chi \gamma}{\sin ^{2} \theta}\right) d \theta \\
& \left.-\frac{8}{M} \sum_{t=1}^{v-1} \frac{1}{\pi} \int_{0}^{\beta_{t}^{-}}-\frac{8 \chi}{\sin ^{2} \theta} \exp \left(\frac{-8 \chi \gamma}{\sin ^{2} \theta}\right) d \theta\right] .
\end{aligned}
$$

Substituting (40) along with (20) in (31), generalized XQAM expression in integral form can be written as

$$
\begin{aligned}
\mathcal{P}_{e}^{X} & =\left[\frac{\left(g_{1}-g_{2}\right)}{2} \sqrt{\frac{\chi}{\pi}} \mathbb{F}\left(\frac{1}{2}, \chi\right)+\frac{4}{M} \sqrt{\frac{\chi}{2 \pi}} \mathbb{F}\left(\frac{1}{2}, 2 \chi\right)+g_{2} \frac{\chi}{\pi} \sum_{z=0}^{\infty} \frac{(1)_{z} \chi^{z}}{z !(1.5)_{z}} \mathbb{F}(z+1,2 \chi)\right]-C_{1} C_{2}^{-2(m+j)} \Gamma(m+j) \\
& \times\left[\frac{\left(g_{1}-g_{2}\right)}{2} \sqrt{\frac{\chi}{\pi}} \mathbb{G}\left(\frac{1}{2}, \chi\right)+\frac{4}{M} \sqrt{\frac{\chi}{2 \pi}} \mathbb{G}\left(\frac{1}{2}, 2 \chi\right)+g_{2} \frac{\chi}{\pi} \sum_{z=0}^{\infty} \frac{(1)_{z} \chi^{z}}{z !(1.5)_{z}} \mathbb{G}(z+1,2 \chi)\right]+A C_{1} \sum_{z_{1}=0}^{m-1+j}\left(\begin{array}{c}
m-1+j \\
z_{1}
\end{array}\right) \\
& \times C_{2}^{-\left(m+j+z_{1}+1\right)}\left[\frac{\left(g_{1}-g_{2}\right)}{2} \sqrt{\frac{\chi}{\pi}} \mathbb{H}\left(\frac{1}{2}, \chi\right)+\frac{4}{M} \sqrt{\frac{\chi}{2 \pi}} \mathbb{H}\left(\frac{1}{2}, 2 \chi\right)+g_{2} \frac{\chi}{\pi} \sum_{z=0}^{\infty} \frac{(1)_{z} \chi^{z}}{z !(1.5)_{z}} \mathbb{H}(z+1,2 \chi)\right] \\
& +\frac{16}{M} \sum_{t=1}^{v-1}\left[\frac { 1 } { \pi } \int _ { 0 } ^ { \infty } \left\{\left(1-C_{1} C_{2}^{-2(m+j)} \Gamma(m+j) \Gamma\left(m+j, C_{2} \gamma\right)+C_{1} A \sum_{z_{1}=0}^{m-1+j}\left(\begin{array}{c}
m-1+j \\
z_{1}
\end{array}\right) C_{2}^{-\left(m+j+z_{1}+1\right)}\right.\right.\right. \\
& \left.\left.\left.\gamma^{m-1+j-z_{1}} e^{-C_{2} \gamma} \mathrm{G}_{i+2,3 i+2}^{3 i+1,2}\left[\left.\frac{B \gamma}{\Omega_{i}}\right|_{1+z_{1}, \mu_{2}, 0} ^{1-(m+j), 1, \mu_{1}}\right]\right) \int_{0}^{\alpha_{t}^{+}}-\frac{\chi}{\sin ^{2} \theta} e^{\frac{-\chi \gamma}{\sin ^{2} \theta}} d \theta\right\} d \gamma\right] \\
& +\frac{8}{M} \sum_{t=1}^{v-1}\left[\frac { 1 } { \pi } \int _ { 0 } ^ { \infty } \left\{\left(1-C_{1} C_{2}^{-2(m+j)} \Gamma(m+j) \Gamma\left(m+j, C_{2} \gamma\right)+C_{1} A \sum_{z_{1}=0}^{m-1+j}\left(\begin{array}{c}
m-1+j \\
z_{1}
\end{array}\right) C_{2}^{-\left(m+j+z_{1}+1\right)}\right.\right.\right. \\
& \left.\left.\left.\gamma^{m-1+j-z_{1}} e^{-C_{2} \gamma} \mathrm{G}_{i+2,3 i+2}^{3 i+1,2}\left[\left.\frac{B \gamma}{\Omega_{i}}\right|_{1+z_{1}, \mu_{2}, 0} ^{1-(m+j), 1, \mu_{1}}\right]\right) \int_{0}^{\beta_{t}^{+}}-\frac{2 \chi}{\sin ^{2} \theta} e^{\frac{-2 \chi \gamma}{\sin ^{2} \theta}} d \theta\right\} d \gamma\right] \\
& -\frac{8}{M} \sum_{t=1}^{v-1}\left[\frac { 1 } { \pi } \int _ { 0 } ^ { \infty } \left\{\left(1-C_{1} C_{2}^{-2(m+j)} \Gamma(m+j) \Gamma\left(m+j, C_{2} \gamma\right)+C_{1} A \sum_{z_{1}=0}^{m-1+j}\left(\begin{array}{c}
m-1+j \\
z_{1}
\end{array}\right) C_{2}^{-\left(m+j+z_{1}+1\right)}\right.\right.\right. \\
& \left.\left.\left.\gamma^{m-1+j-z_{1}} e^{-C_{2} \gamma} \mathrm{G}_{i+2,3 i+2}^{3 i+1,2}\left[\left.\frac{B \gamma}{\Omega_{i}}\right|_{1+z_{1}, \mu_{2}, 0} ^{1-(m+j), 1, \mu_{1}}\right]\right) \int_{0}^{\beta_{t}^{-}}-\frac{8 \chi}{\sin ^{2} \theta} e^{\frac{-8 \chi \gamma}{\sin ^{2} \theta}} d \theta\right\} d \gamma\right] .
\end{aligned}
$$

Considering the limiting case of generalized XQAM for $M=32,(v=1)$, only the starting 3 terms of conditional SEP expression (38) are considered as last 3 terms will be zero for $v=1$. Hence, closed-form ASER expression for 32-XQAM is derived as

$$
\begin{aligned}
\mathcal{P}_{e}^{X} & =\left[\frac{g_{1}-g_{2}}{2} \sqrt{\frac{\chi}{\pi}} \mathbb{F}\left(\frac{1}{2}, \chi\right)+\frac{4}{M} \sqrt{\frac{\chi}{2 \pi}} \mathbb{F}\left(\frac{1}{2}, 2 \chi\right)+g_{2} \frac{\chi}{\pi} \sum_{z=0}^{\infty} \frac{(1)_{z} \chi^{z}}{z !(1.5)_{z}} \mathbb{F}(z+1,2 \chi)\right] \\
& -C_{1} C_{2}^{-2(m+j)} \Gamma(m+j)\left[\frac{g_{1}-g_{2}}{2} \sqrt{\frac{\chi}{\pi}} \mathbb{G}\left(\frac{1}{2}, \chi\right)+\frac{4}{M} \sqrt{\frac{\chi}{2 \pi}} \mathbb{G}\left(\frac{1}{2}, 2 \chi\right)\right. \\
& \left.+g_{2} \frac{\chi}{\pi} \sum_{z=0}^{\infty} \frac{(1)_{z} \chi^{z}}{z !(1.5)_{z}} \mathbb{G}(z+1,2 \chi)\right]+A C_{1} \sum_{z_{1}=0}^{m-1+j}\left(\begin{array}{c}
m-1+j \\
z_{1}
\end{array}\right) C_{2}^{-\left(m+j+z_{1}+1\right)} \\
& \times\left[\frac{g_{1}-g_{2}}{2} \sqrt{\frac{\chi}{\pi}} \mathbb{H}\left(\frac{1}{2}, \chi\right)+\frac{4}{M} \sqrt{\frac{\chi}{2 \pi}} \mathbb{H}\left(\frac{1}{2}, 2 \chi\right)+g_{2} \frac{\chi}{\pi} \sum_{z=0}^{\infty} \frac{(1)_{z} \chi^{z}}{z !(1.5)_{z}} \mathbb{H}(z+1,2 \chi)\right] .
\end{aligned}
$$

\section{Theoretical And Simulation Results}

In this Section, theoretical result are numerically evaluated and validated through simulations. RF link is modeled with Nakagami-m distributions and $m=1,2$ are considered to show the impact of fading on system's performance. FSO link is modeled with the atmospheric turbulence influenced Gamma-Gamma distribution with pointing error. Both IM/DD and heterodyne detection are employed at the FSO receiver [13]. For analysis, atmospheric turbulence parameters $\alpha=2.1, \beta=3.5$ 
are considered. $\xi=1$ for severe pointing error and $\xi=6.7$ for negligible pointing error are considered. Outdated CSI is considered at the relay, and various values of correlation coefficient $\rho$ are considered for the analysis.

As an infinite series presents in the derived analytical outage probability, ergodic capacity, and ASER results, the infinite summation series $j$ is truncated to 100 summation terms for better numerical accuracy with considerable implementation complexity [8]. Summation terms may be further reduced for lower value of $\rho$.

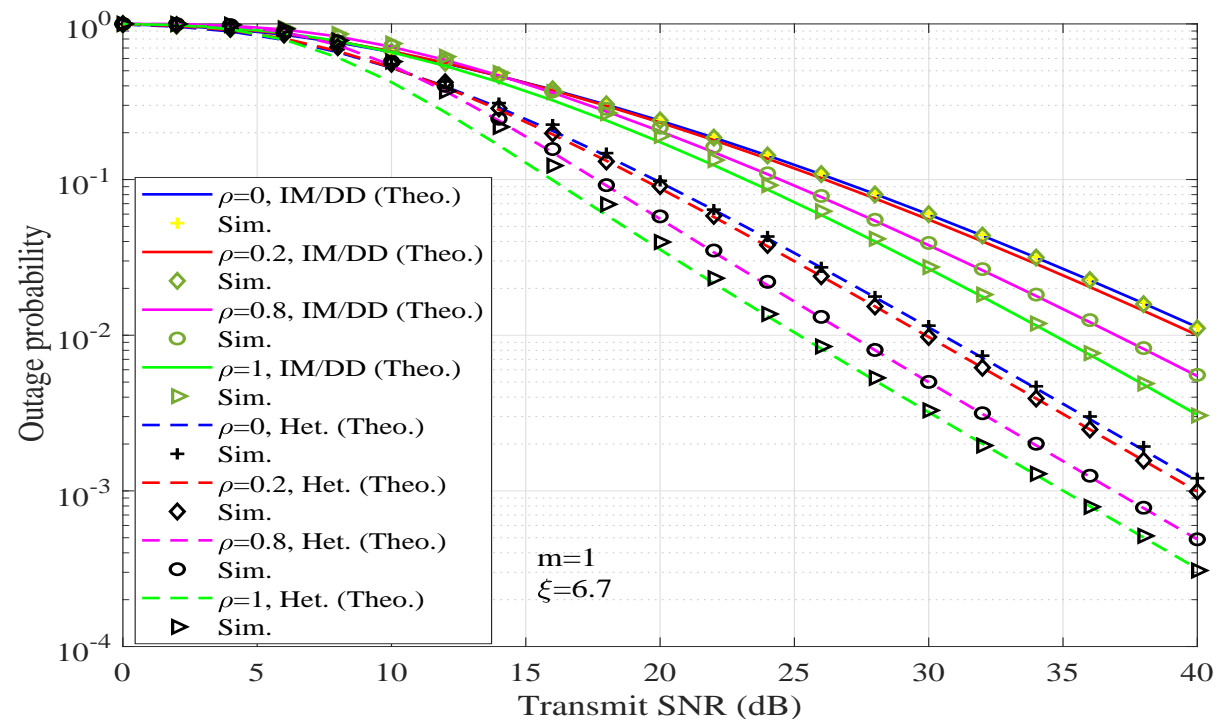

Fig. 3: Theoretical and simulation results of outage probability for $\alpha=2.1$ and $\beta=3.5$.

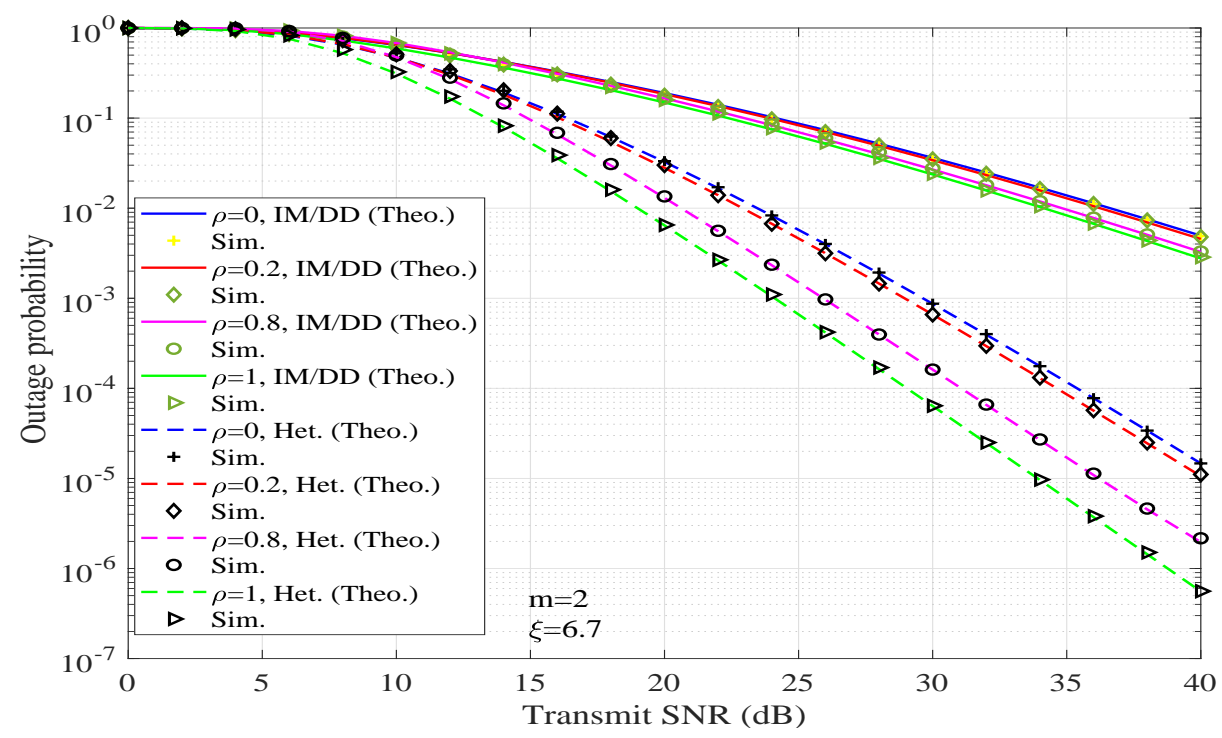

Fig. 4: Theoretical and simulation results of outage probability for $\alpha=2.1$ and $\beta=3.5$.

In Fig. 3 and Fig. 4, theoretical (Theo.) and simulation (Sim.) results of outage probability for $m=1$ and $m=2$, respectively are compared for various values of $\rho(\rho=0,0.2,0.8,1)$ and $\xi=6.7$. Both the corner cases, i.e. $\rho=0$ (uncorrelated CSI) and $\rho=1$ (perfectly correlated) are also considered. With the increase in $\rho$ from 0 to 1 , improvement in outage performance is observed. Further, heterodyne detection provides better outage performance than IM/DD detection. This improvement is due the fact that the turbulence effects are better handled by heterodyne technique [13]. However, this can be achieved with increased implementation complexity as compared to the IM/DD technique. Simulation results match well with the theoretical results and also validate the correctness of the derived expressions. For the perfect CSI case $(\rho=1)$, upper-bound of e2e SNR is considered and hence, lower-bound of the outage probability is obtained. Thus, theoretical results are slightly below the simulation results, matches perfectly in medium and high SNR regime.

In Fig. 5, outage probability is compared for severe pointing error $(\xi=1)$ and negligible pointing error $(\xi=6.7)$ cases while various values of $m$ and both the IM/DD and heterodyne detections are considered. It can be observed, for both the detection techniques, outage performance is significantly deteriorated for $\xi=1$ as compared to $\xi=6.7$ and comparatively less 


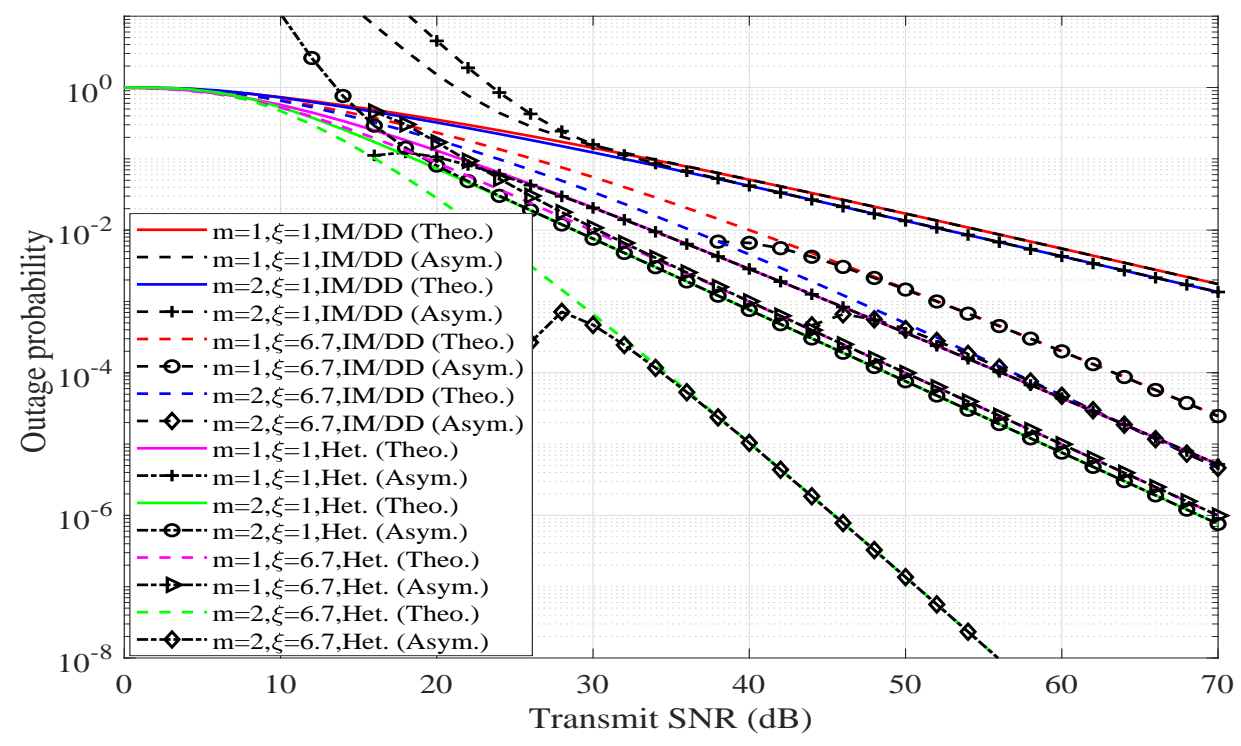

Fig. 5: Theoretical and asymptotic results of outage probability for $\alpha=2.1$ and $\beta=3.5$.

improvement in outage performance in observed with the increase in $m$. Asymptotic outage curves (Asym.) are also shown in Fig. 5, which matches well at high SNRs for all the investigated cases and validate the high SNR analysis. As a mixed RF/FSO system is considered, the diversity order of the considered system is $\min \left(m, \frac{\xi^{2}}{i}, \frac{\alpha}{i}, \frac{\beta}{i}\right)$, which depends on the fading severity of RF link $m$, pointing error $\xi$, and turbulence parameters $\alpha$ and $\beta$. This can also be validated through the results shown in Fig. 5, that the diversity order varies with $\xi$. Further, correlation coefficient $\rho$ have no impact on the diversity order of the considered system. The slope of the outage curves remain same with the change in $\rho$ as illustrated in Fig. 3 and Fig. 4, however, considerable SNR improvement is observed with the increase in $\rho$.

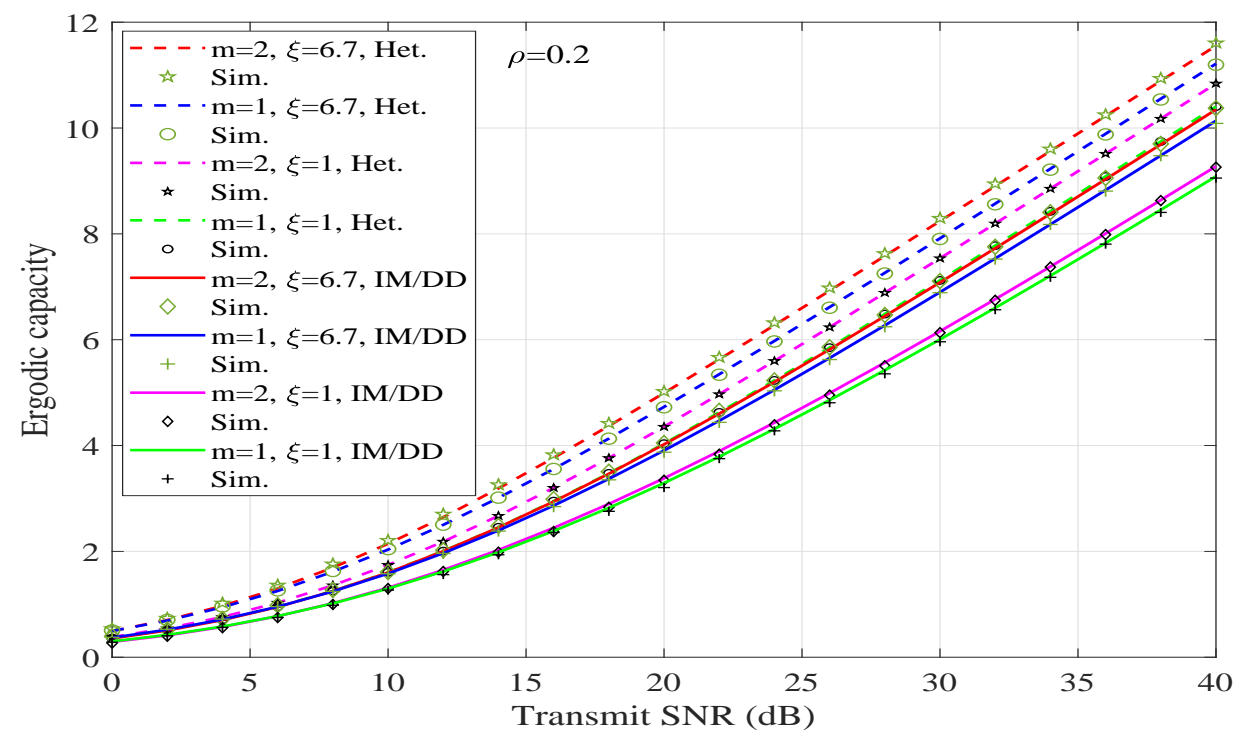

Fig. 6: Comparison of theoretical and simulation results of ergodic capacity for $\alpha=2.1, \beta=3.5$ and $\rho=0.2$.

In Fig. 6, theoretical and simulation results of ergodic capacity are presented for both the IM/DD and heterodyne detection under severe pointing error $(\xi=1)$ and negligible pointing error $(\xi=6.7)$ cases. For analysis, fixed values of $\alpha=2.1, \beta=3.5$ and $\rho=0.2$ are considered. It can be described that the ergodic capacity decreases considerably when $\xi=1$ is considered as compared to $\xi=6.7$ for both the detection techniques. Heterodyne detection provides improved performance than IM/DD for both the severe and negligible pointing error cases despite its detection complexity. Further, ergodic capacity improves with the increase in $m$, however, slightly less improvement is observed in IM/DD than in heterodyne detection.

In Fig. 7, theoretical and simulation results of ASER of 16-HQAM are presented for different values of $\rho$ with heterodyne detection. For analysis, fixed values of $\alpha=2.1, \beta=3.5$ and $\xi=6.7$ are considered. From Fig. 7, it is observed that the simulation results validate correctness of the theoretical results. Further, ASER results improves as $\rho$ increases from 0 to 1 (uncorrelated to perfectly correlated case). Furthermore, significant ASER performance improvement is achieved with the increase in $m$ from 1 to 2. 


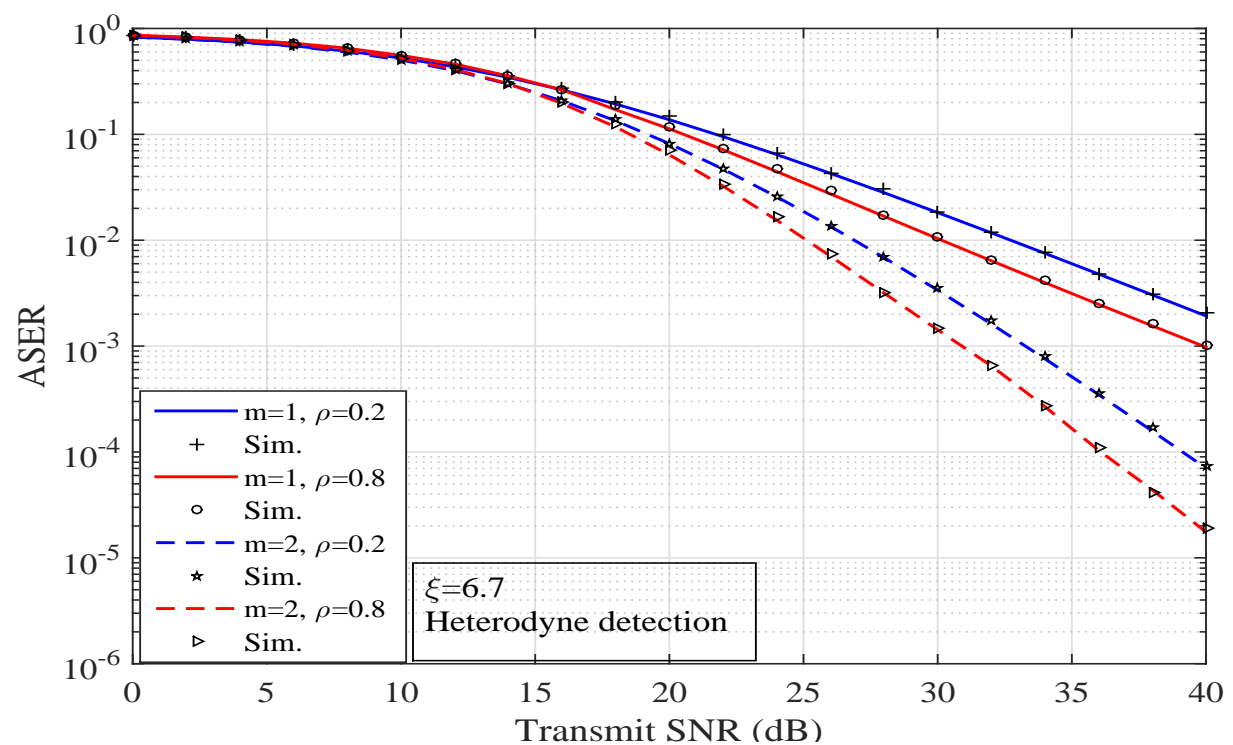

Fig. 7: Comparison of theoretical and simulation results of 16-HQAM for $\alpha=2.1, \beta=3.5$ and different values of $\rho$.

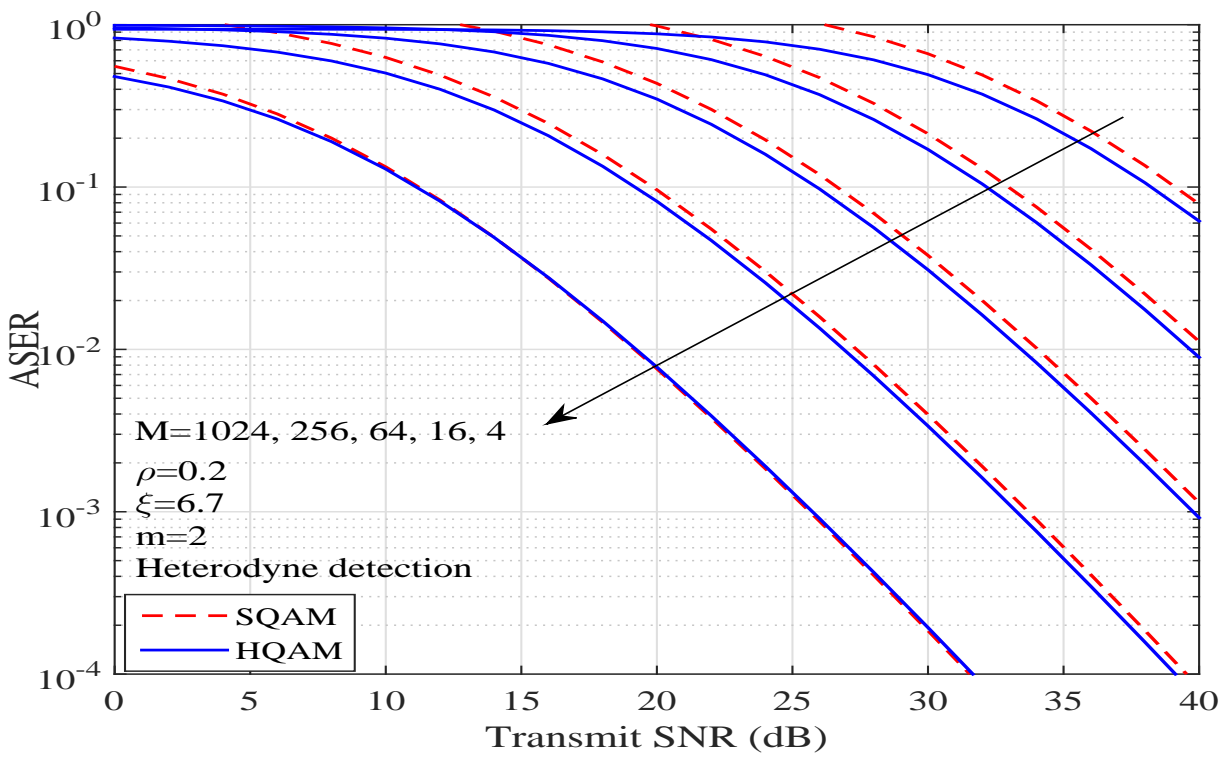

Fig. 8: Comparison of theoretical results of HQAM and SQAM schemes for constellations of even power of 2 with $\alpha=2.1, \beta=3.5$.

In Fig. 8, Fig. 9, and Fig. 10, theoretical ASER results of HQAM are presented which are compared with various QAM techniques. For the analysis, $\rho=0.2, \xi=6.7$ and $m=1$ are considered. In Fig. 8, only the even power of 2 constellations are considered and HQAM results are compared with the SQAM results. To achieve ASER of $10^{-2}$, 4-SQAM provides around $0.065 \mathrm{~dB}$ gain over the 4-HQAM schemes. However, for increasing values of M, HQAM outperforms the SQAM scheme as can be seen from Fig. 8. To achieve $10^{-2}$ ASER, respective gain of around $0.475 \mathrm{~dB}, 0.612 \mathrm{~dB}$ and $0.664 \mathrm{~dB}$ are achieved for HQAM scheme over the SQAM scheme for 16, 64 and 256 constellation points. We observe that the slope difference in HQAM and SQAM schemes for various constellations are approximately same after $10^{-1}$ ASER, hence, SNR gain for 1024 points constellations of HQAM over SQAM scheme is achieved at $10^{-1}$ ASER. It can be observed that the SNR gains of around $0.89 \mathrm{~dB}$ is achieved in HQAM over the SQAM scheme for 1024 points constellations. This justifies the superiority of HQAM over the SQAM schemes and gain improves further for increasing constellation orders. In Fig. 9, odd power of 2 constellations are considered. Theoretical results of HQAM scheme for $8,32,128$ point constellations are presented and a comparative study with $4 \times 2$-RQAM, $8 \times 4$-RQAM, 32-XQAM, $16 \times 8$-RQAM and 128-XQAM schemes is presented for $m=1$ and $m=2$. For the analysis, fixed values of $\alpha=2.1, \beta=3.5, \rho=0.2$ and $\xi=6.7$ are considered. From Fig. 9, it is observed that to achieve $10^{-2}$ ASER for $m=2$, around $1 \mathrm{~dB}$ gain is received for 8 -HQAM over $4 \times 2$-RQAM scheme. Further, 32-HQAM provides around $1.2 \mathrm{~dB}$ and $0.13 \mathrm{~dB}$ gains over $8 \times 4$-RQAM and 32-XQAM schemes, respectively. Similarly, 128-HQAM provides around $1.30 \mathrm{~dB}$ and $0.20 \mathrm{~dB}$ gains over $16 \times 8$-RQAM and 128-XQAM schemes, respectively. From this, it can be concluded that irregular HQAM is the optimum modulation scheme which provides considerable SNR gain over 


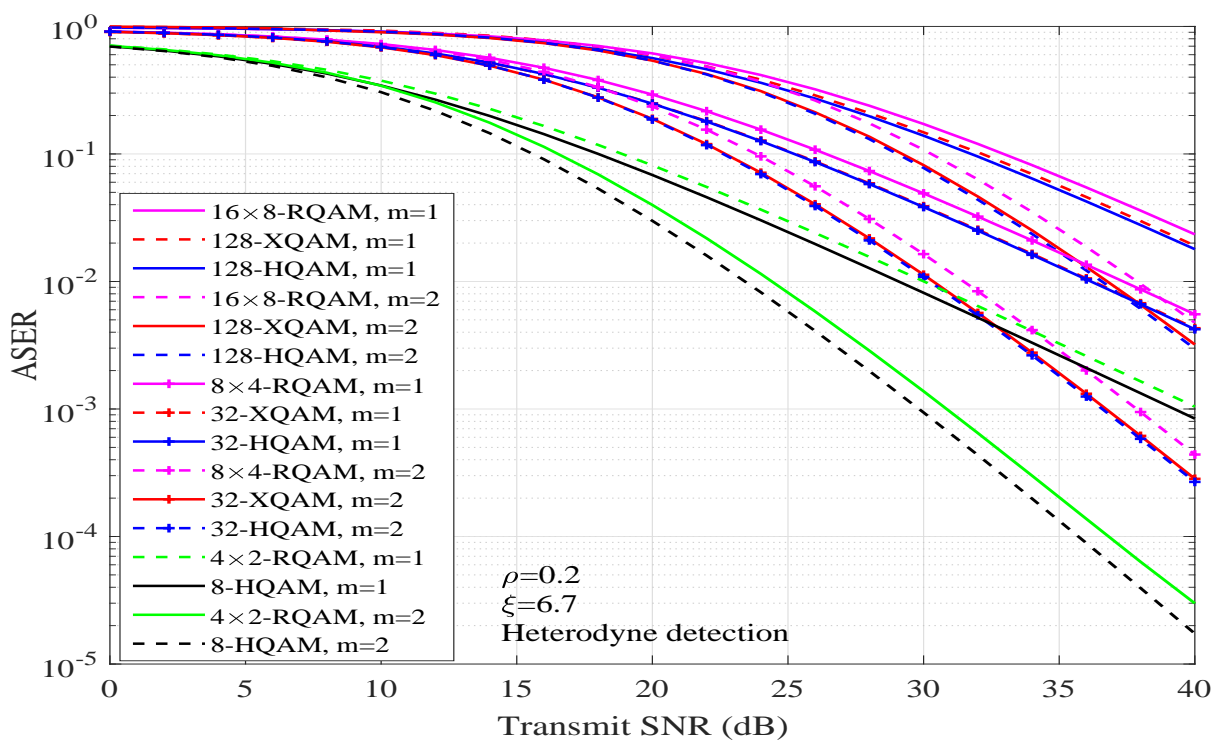

Fig. 9: Comparison of theoretical results of HQAM, XQAM and RQAM schemes for constellations of odd power of 2 with $\alpha=2.1$, $\beta=3.5$.

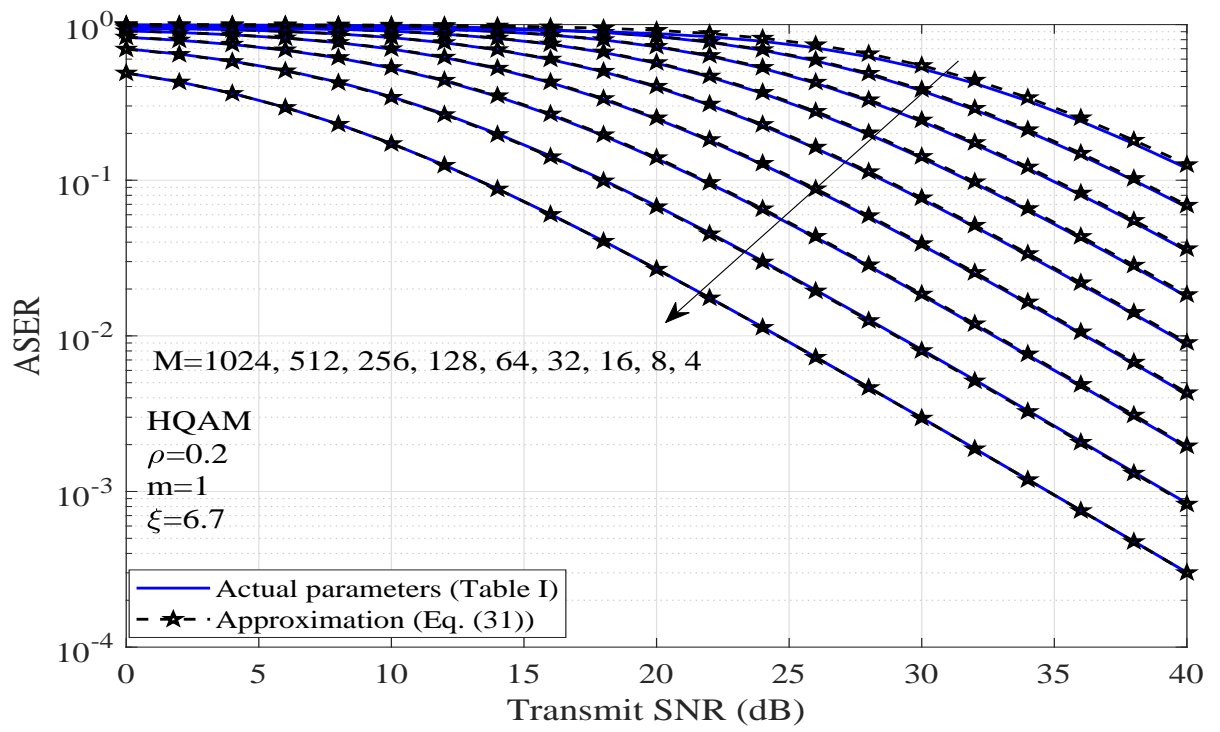

Fig. 10: Comparative study of ASER results of various HQAM constellations for exact and approximate values of $K, \tau$ and $\tau_{c}$.

the other modulation schemes, and this gain improves further for the increasing constellation orders. In (34), generalization for $K, \tau$, and $\tau_{c}$ is performed and Fig. 10 illustrates a comparative study of ASER results of various HQAM constellations for exact and approximate values of $K, \tau$, and $\tau_{c}$ as given in Table I and (34), respectively. For analysis, $\alpha=2.1, \beta=3.5$, $\xi=6.7, \rho=0.2$ and $m=1$ are considered. It is observed that the approximate results match well with the exact ones for all the HQAM constellations up to 1024 which validates the generalization of $K, \tau$, and $\tau_{c}$ as given in (34).

\section{CONCLUSIONS}

In this work, performance of a variable gain dual-hop AF mixed RF/FSO system for both the IM/DD and heterodyne detection has been analyzed. Outdated CSI in the RF part and atmospheric turbulence influenced FSO link with pointing error have been considered. Exact analytical expressions of outage probability and ergodic capacity in terms of Meijer-G function and EGBMGF have been derived, and impact of outdated CSI, pointing error, fading parameter and type of detection have been shown. It has been concluded that despite detection complexity, heterodyne detection provides better outage performance, and ergodic capacity than IM/DD. Asymptotic outage probability has also been derived to find out the diversity order of the considered system. Various higher order optimum irregular HQAM constellations have been presented and CDF based exact ASER expressions of general order HQAM, XQAM, and RQAM schemes in terms of Meijer-G function have been derived. A comparative study of various QAM schemes has been presented which illustrates the superiority of irregular HQAM constellations over the other QAM schemes. 


\section{REFERENCES}

[1] M. Abdelaziz and T. A. Gulliver, "Triangular constellations for adaptive modulation," IEEE Trans. Commun., vol. 66, no. 2, pp. 756-766, Feb. 2018.

[2] N. Kumar, P. K. Singya, and V. Bhatia, "ASER analysis of hexagonal and rectangular QAM schemes in multiple-relay networks," IEEE Trans. Veh. Technol., vol. 67, no. 2, pp. 1815-1819, Feb. 2018.

[3] P. K. Singya, N. Kumar, and V. Bhatia, "Impact of imperfect CSI on ASER of hexagonal and rectangular QAM for AF relaying network," IEEE Commun. Lett., vol. 22, no. 2, pp. 428-431, Feb. 2018.

[4] P. K. Singya, N. Kumar, V. Bhatia, and M.-S. Alouini, "On performance of hexagonal, cross, and rectangular QAM for multi-relay systems," IEEE Access, vol. 7, pp. 60602-60616, May 2019.

[5] W. T. Webb, "QAM: the modulation scheme for future mobile radio communications?" IET Electron. Commun. Engg. J., vol. 4, no. 4, pp. 167-176, Aug. 1992.

[6] P. Yang, Y. Xiao, B. Zhang, S. Li, M. El-Hajjar, and L. Hanzo, "Star-QAM signaling constellations for spatial modulation," IEEE Trans. Veh. Technol., vol. 63, no. 8, pp. 3741-3749, Feb. 2014.

[7] L. Yu, P. Fan, D. Cai, and Z. Ma, "Design and analysis of SCMA codebook based on star-QAM signaling constellations," IEEE Trans. Veh. Technol., vol. 67, no. 11, pp. 10543-10553, Aug. 2018.

[8] G. T. Djordjevic, M. I. Petkovic, A. M. Cvetkovic, and G. K. Karagiannidis, "Mixed RF/FSO relaying with outdated channel state information," IEEE J. Selected Areas Commun., vol. 33, no. 9, pp. 1935-1948, Sep. 2015.

[9] E. Lee, J. Park, D. Han, and G. Yoon, "Performance analysis of the asymmetric dual-hop relay transmission with mixed RF/FSO links," IEEE Photon Technol. Lett., vol. 23, no. 21, pp. 1642-1644, Nov. 2011.

[10] I. S. Ansari, F. Yilmaz, and M.-S. Alouini, "Impact of pointing errors on the performance of mixed RF/FSO dual-hop transmission systems," IEEE Wireless Commun. Lett., vol. 2, no. 3, pp. 351-354, Jun. 2013.

[11] H. Samimi and M. Uysal, "End-to-end performance of mixed RF/FSO transmission systems," IEEE/OSA J. Optical Commun. Netw., vol. 5, no. 11, pp. 1139-1144, Nov. 2013

[12] E. Zedini, I. S. Ansari, and M.-S. Alouini, "Performance analysis of mixed Nakagami- $m$ and Gamma-Gamma dual-hop FSO transmission systems," IEEE Photon. J., vol. 7, no. 1, pp. 1-20, Dec. 2014.

[13] E. Zedini, H. Soury, and M.-S. Alouini, "On the performance analysis of dual-hop mixed FSO/RF systems," IEEE Trans. Wireless Commun., vol. 15, no. 5, pp. 3679-3689, May 2016.

[14] J. Chen, L. Yang, W. Wang, H.-C. Yang, Y. Liu, M. O. Hasna, and M.-S. Alouini, "A novel energy harvesting scheme for mixed FSO-RF relaying systems," IEEE Trans. Veh. Technol., vol. 68, no. 8, pp. 8259-8263, Aug. 2019.

[15] L. Yang, T. Liu, J. Chen, and M.-S. Alouini, "Physical-layer security for mixed $\eta-\mu$ and $\mathcal{M}$-distribution dual-hop RF/FSO systems," IEEE Trans. Veh. Technol., vol. 67, no. 12, pp. 12427-12431, Oct. 2018.

[16] E. Soleimani-Nasab and M. Uysal, "Generalized performance analysis of mixed RF/FSO cooperative systems," IEEE Trans. Wireless Commun., vol. 15, no. 1, pp. 714-727, Sep. 2015.

[17] L. Yang, M. O. Hasna, and I. S. Ansari, "Unified performance analysis for multiuser mixed $\eta-\mu$ and $\mathcal{M}$-distribution dual-hop RF/FSO systems," IEEE Trans. Commun., vol. 65, no. 8, pp. 3601-3613, May 2017.

[18] H. Arezumand, H. Zamiri-Jafarian, and E. Soleimani-Nasab, "Outage and diversity analysis of underlay cognitive mixed RF-FSO cooperative systems," IEEE/OSA J. Optical Commun. Netw., vol. 9, no. 10, pp. 909-920, 2017.

[19] E. Balti, M. Guizani, B. Hamdaoui, and B. Khalfi, "Aggregate hardware impairments over mixed RF/FSO relaying systems with outdated CSI," IEEE Trans. Commun., vol. 66, no. 3, pp. 1110-1123, Mar. 2018.

[20] M. I. Petkovic, A. M. Cvetkovic, G. T. Djordjevic, and G. K. Karagiannidis, "Partial relay selection with outdated channel state estimation in mixed RF/FSO systems," J. Lightwave Technol., vol. 33, no. 13, pp. 2860-2867, Jul. 2015.

[21] D. Dixit and P. R. Sahu, "Performance analysis of rectangular QAM with SC receiver over Nakagami-m fading channels," IEEE Commun. Lett., vol. 18, no. 7, pp. 1262-1265, Jul. 2014.

[22] J. P. Peña-Martín, J. M. Romero-Jerez, and C. Tellez-Labao, "Performance of selection combining diversity in $\eta-\mu$ fading channels with integer values of $\mu$," IEEE Trans. Veh. Technol., vol. 64, no. 2, pp. 834-839, Feb. 2015.

[23] L. Rugini, "Symbol error probability of hexagonal QAM," IEEE Commun. Lett., vol. 20, no. 8, pp. 1523-1526, Aug. 2016.

[24] P. K. Singya, N. Kumar, and V. Bhatia, "Performance analysis of AF OFDM system using multiple relay in presence of nonlinear-PA over inid Nakagami-m fading," Wiley Int. J. Commun. Syst., vol. 31, no. 1, Jan. 2018.

[25] P. Shaik, P. K. Singya, and V. Bhatia, "On impact of imperfect CSI over hexagonal QAM for TAS/MRC-MIMO cooperative relay network," IEEE Commun. Lett., vol. 23, no. 10, pp. 1721-1724, Jul. 2019.

[26] S. J. Park, "Performance analysis of triangular quadrature amplitude modulation in AWGN channel," IEEE Commun. Lett., vol. 16, no. 6, pp. 765-768, Jun. 2012.

[27] S. Park and M.-K. Byeon, "Irregularly distributed triangular quadrature amplitude modulation," in IEEE 19th Int. Symp. Personal, Indoor Mobile Radio Commun., Sep. 2008, pp. 1-5.

[28] S. Park, M. Byeon, and J. Jeon, "Odd-bit triangular quadrature amplitude modulations," in IEEE 20th Int. Symp. Personal, Indoor Mobile Radio Commun., Sep. 2009, pp. 2419-2423.

[29] I. S. Ansari, F. Yilmaz, and M.-S. Alouini, "Performance analysis of free-space optical links over Malaga turbulence channels with Pointing errors," IEEE Trans. Wireless Commun., vol. 15, no. 1, pp. 91-102, Jan. 2016.

[30] E. Zedini, I. S. Ansari, and M.-S. Alouini, "On the performance of hybrid line of sight RF and RF-FSO fixed gain dual-hop transmission systems," in IEEE Global Commun. Conf. IEEE, Dec. 2014, pp. 2119-2124.

[31] M.-S. Alouini and A. J. Goldsmith, "Adaptive modulation over Nakagami fading channels," Wireless Personal Commun., vol. 13, no. 1-2, pp. 119-143, May 2000

[32] N. S. Ferdinand, D. B. da Costa, and M. Latva-aho, "Effects of outdated CSI on the secrecy performance of MISO wiretap channels with transmit antenna selection," IEEE Commun. Lett., vol. 17, no. 5, pp. 864-867, May 2013.

[33] I. Gradshteyn and I. Ryzhik, Table of Integrals, Series and Products. 6th ed. New York, NY, USA: Academic, 2000.

[34] The Wolfram Function Site. [Online]. Available: http://functions.wolfram.com/

[35] I. S. Ansari, S. Al-Ahmadi, F. Yilmaz, M.-S. Alouini, and H. Yanikomeroglu, "A new formula for the BER of binary modulations with dual-branch selection over generalized-K composite fading channels," IEEE Trans. Commun., vol. 59, no. 10, pp. 2654-2658, Oct. 2011

[36] G. Forney, R. Gallager, G. Lang, F. Longstaff, and S. Qureshi, "Efficient modulation for band-limited channels," IEEE J. Selected Areas Commun., vol. 2, no. 5, pp. 632-647, Sep. 1984.

[37] M. Abramowitz and I. A. Stegun, Handbook of Mathematical Functions: with formulas, graphs, and mathematical tables. 9th ed. New York, NY, USA: Dover, 1970.

[38] X.-C. Zhang, H. Yu, and G. Wei, "Exact symbol error probability of cross-QAM in AWGN and fading channels," EURASIP J. Wireless Commun. Netw., vol. 2010, p. 94, 2010. 